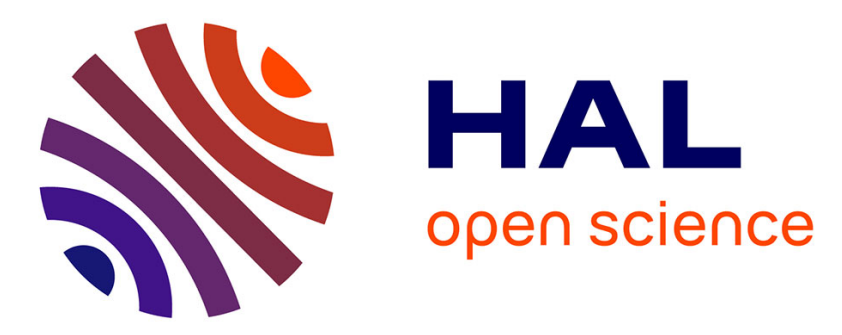

\title{
Nonparametric stochastic modeling of structures with uncertain boundary conditions / coupling between substructures
}

\author{
M. P. Mignolet, Christian Soize, J. Avalos
}

\section{- To cite this version:}

M. P. Mignolet, Christian Soize, J. Avalos. Nonparametric stochastic modeling of structures with uncertain boundary conditions / coupling between substructures. AIAA Journal, 2013, 51 (6), pp.12961308. 10.2514/1.J051555 . hal-00803746

\section{HAL Id: hal-00803746 \\ https://hal.science/hal-00803746}

Submitted on 22 Mar 2013

HAL is a multi-disciplinary open access archive for the deposit and dissemination of scientific research documents, whether they are published or not. The documents may come from teaching and research institutions in France or abroad, or from public or private research centers.
L'archive ouverte pluridisciplinaire HAL, est destinée au dépôt et à la diffusion de documents scientifiques de niveau recherche, publiés ou non, émanant des établissements d'enseignement et de recherche français ou étrangers, des laboratoires publics ou privés. 


\title{
Nonparametric Stochastic Modeling of Structures with Uncertain Boundary Conditions / Coupling Between Substructures
}

\author{
Marc P. Mignolet ${ }^{1}$ \\ Arizona State University, Tempe, AZ, 85287-6106, USA. \\ Christian Soize ${ }^{2}$ \\ Université Paris-Est, Marne-la-Vallee, 77454, France.
}

Javier Avalos ${ }^{3}$

Instituto Technológico y de Estudios Superiores de Occidente (ITESO), Tlaquepaque, Jalisco, 45604Mexico.

The focus of this investigation is on the formulation and validation of a novel approach for the inclusion of uncertainty in the modeling of the boundary conditions of linear structures and of the coupling between linear substructures. This work is particularly relevant to complex structures assembled from simpler substructures as in aerospace applications. First, a mean structural dynamic model that includes boundary condition/coupling flexibility is obtained using classical substructuring concepts. The application of the nonparametric stochastic modeling approach to this mean model is next described and thus permits the consideration of both model and parameter uncertainty. Finally, a dedicated identification procedure is proposed to estimate the two parameters of this stochastic model, i.e. the mean boundary condition/coupling flexibility and the overall level of uncertainty. The methodology is demonstrated on three different structural dynamic models, i.e. of a rectangular plate and of two different wings.

\section{Nomenclature}

$\delta=$ overall measure of uncertainty

$k \quad=\quad$ scalar parameter of the boundary condition modeling

$\boldsymbol{K}_{\text {phys }} \quad=$ structure stiffness matrix

$\boldsymbol{K}_{C B} \quad=$ Craig-Bampton stiffness matrix

$\boldsymbol{K}_{R O M}=$ Reduced Order Model stiffness matrix

$\boldsymbol{X}_{\text {phys }}^{I} \quad=$ internal degrees of freedom vector

${ }^{1}$ Professor, SEMTE, Faculties of Mechanical and Aerospace Engineering, marc.mignolet@asu.edu, Associate Fellow, AIAA.

${ }^{2}$ Professor, Laboratoire Modélisation et Simulation Multi Echelle, christian.soize@univ-paris-est.fr

${ }^{3}$ Assistant Professor, Ingeniería Mecánica, javalos@iteso.mx, member AIAA. 


$$
\begin{array}{ll}
\boldsymbol{X}_{\text {phys }}^{B} & =\text { boundary degrees of freedom vector } \\
\boldsymbol{Y} & =\text { boundary degrees of freedom } \\
\boldsymbol{q} & =\text { modal internal generalized coordinates vector } \\
\boldsymbol{u} & =\text { modal boundary generalized coordinates vector } \\
\boldsymbol{\Phi} & =\text { clamped structure mode shapes } \\
\boldsymbol{\Xi} & =\text { constraint modes } \\
\Psi & =\text { boundary mode shapes } \\
E_{B C} & =\text { boundary condition energy }
\end{array}
$$

\section{Introduction}

Significant efforts have been focused in last decade or so on the modeling and consideration of uncertainty in the properties of structural dynamic systems. In fact, two types of uncertainty have been recognized, see [1] for discussion. Parameter uncertainty refers to a lack of knowledge of the exact values of the parameters of the physical and/or computational model, e.g. of the Young's modulus, see [2-5]. Model uncertainty on the other hand relates to discrepancies between the physical structure and its model that arise in the modeling effort, e.g. in the finite element representation of the connection between two parts by rivets, spot welds, etc.

Parameter uncertainties can be considered straightforwardly in full order (e.g. finite element) models through the introduction of random variables/stochastic processes that describe the uncertain parameters. The parameter uncertainty effects will then be estimated from a Monte Carlo analysis of a single full order model with different parameter values. Note that the identification of the statistical description of the parameters (e.g. selection of their joint probability density functions) is often a challenge owing to the generally limited information on their uncertainty/variability.

The treatment of model uncertainty is typically much more complex and may require the construction of an ensemble of different full order models. A convenient alternative to full order models are reduced order (modal) models in which the basis functions (modes) are fixed/deterministic. Not only are these models computationally much faster than their full order counterparts, a desirable feature when performing Monte Carlo analyses, but they also provide a unified framework for addressing both model and parameter uncertainties which are regrouped into the mass and stiffness matrices. The consideration of uncertainty in this framework is thus reduced to the simulation of random stiffness and/or mass matrices that are compatible with the structural model considered and a particularly efficient and mathematically sound framework to achieve this task is the nonparametric approach, see [1] for review.

Notorious sources of uncertainty in structures are the boundary conditions (especially the clamped ones) and the coupling between substructures which have been found to lead to significant uncertainty in the response of structures exhibiting in particular closely spaced frequencies even in the low frequency domain, e.g. as in bladed disks (see [6]) or in wings near flutter as shown here. Note that both model and parameter uncertainties are in 
general present. Consider for example the clamped boundary condition although a similar discussion can be carried for other boundary conditions and for the coupling between substructures. A first modeling strategy of a physical clamped boundary condition is in terms of its mathematical counterpart, i.e. zero displacements and slopes. This approach however completely neglects the unavoidable flexibility of the support and clamp and thus leads to an overestimation of the natural frequencies. More refined models have then been proposed that do account for this flexibility through the introduction of stiffnesses at the interface between the structure and its support considered rigid, e.g. see [7-10]. Further, uncertainty in these stiffnesses, i.e. parameter uncertainty, has been considered, e.g. see $[9,10]$, to simulate the variability that originates most notoriously from the level of normal force applied at the clamp but also from the state of surface/wear of the structure at its boundary and of the clamp, etc.

While these efforts do capture some of uncertainty in the boundary conditions, they are restricted by the particular set of stiffnesses introduced or the selected form of the boundary stiffness matrix, i.e. they can account for parameter uncertainty but not for model uncertainty. Such uncertainty is however fully expected in this representation of the boundary conditions owing to the complexity of the interactions at the boundary interface (e.g. with contact nonlinearity, possible gaps, friction, etc.) which are only approximated through the introduction of linear stiffness constants.

In this light, the focus of this paper is on the formulation and validation of a novel procedure for the construction of stochastic linear structural dynamic models accounting explicitly for parameter and model uncertainties in boundary conditions and/or coupling between substructures in the low frequency domain. This objective will be achieved by employing the nonparametric approach [1,11-13], briefly reviewed below, which has been shown to address both parameter and model uncertainties as necessary here.

\section{Nonparametric Stochastic Modeling of Uncertainty}

The fundamental problem of the nonparametric approach is the simulation of random symmetric positive definite real matrices $\boldsymbol{A}$ such as the mass, damping, and/or stiffness matrices of linear modal models. To achieve this effort, it is necessary to specify which (joint) statistical distribution of their elements $A_{i j}$ should be adopted. In this regard, it will first be assumed that the mean of the random matrix $\boldsymbol{A}$ is known as $\overline{\boldsymbol{A}}$, i.e. $E[\boldsymbol{A}]=\overline{\boldsymbol{A}}$ where $E[$.] denotes the operation of mathematical expectation. If the fixed modes used to represent the motion of the uncertain structures are those associated with the mean structural model (also referred to as the design conditions model) and are mass normalized, the mean of the random mass and stiffness matrices are the identity matrix and the diagonal matrix of the squared natural frequencies, respectively. Further, if the mean model does not exhibit any rigid body mode (i.e. $\overline{\boldsymbol{A}}$ is strictly positive definite), then it is also expected that the random matrices $\boldsymbol{A}$ will share the same property (note that the extension of the methodology to mean models exhibiting rigid body modes has been accomplished in [12]). This condition is equivalent to the existence of a flat zero at zero of the probability density function of the eigenvalues of $\boldsymbol{A}$. Finally, it will be assumed that only a single measure of the variability of the matrices $\boldsymbol{A}$ is available, e.g. the standard deviation of the lowest eigenvalue of $\boldsymbol{A}$ (the extension of the methodology to account for multiple known measures of variability has been accomplished in [13]). 
Even with the above assumptions (known mean model, nonsingurality of $\boldsymbol{A}$, and known measure of variability), there is a broad set of statistical distributions of the elements $A_{i j}$ that could be selected. Among these, it would be particularly desirable to select the one that places particular emphasis on "larger" deviations from the mean value, a desirable feature to assess, in a limited Monte Carlo study, the aeroelastic robustness of a design to uncertainty. As discussed in [1,11-13], this property arises when the distribution of the elements $A_{i j}$ achieves the maximum of the statistical entropy under the stated constraints of symmetry, positive definiteness, known mean model, nonsingurality of $\boldsymbol{A}$, and known measure of variability. This maximum is obtained (see [1,11-13]) when the matrices $\boldsymbol{A}$ are generated as

$$
\boldsymbol{A}=\overline{\boldsymbol{L}} \boldsymbol{H} \boldsymbol{H}^{T} \overline{\boldsymbol{L}^{T}}
$$

where $\overline{\boldsymbol{L}}$ is any decomposition, e.g. Cholesky, of $\overline{\boldsymbol{A}}$, i.e. satisfying $\overline{\boldsymbol{A}}=\overline{\boldsymbol{L}} \overline{\boldsymbol{L}}^{T}$, the superscript ${ }^{T}$ denoting the operation of matrix transposition. Further, $\boldsymbol{H}$ denotes a lower triangular random matrix the elements which are all statistically independent of each other. Moreover, the probability density functions of the diagonal $\left(H_{i i}\right)$ and offdiagonal elements $\left(H_{i l}\right)$ are

$$
p_{H_{i i}}(h)=C_{i i} h^{p(i)} \exp \left[-\mu h^{2}\right], \quad h \geq 0
$$

and

$$
p_{H_{i l}}(h)=C_{i l} \exp \left[-\mu h^{2}\right], \quad h \geq 0 \quad i \neq l
$$

where

$$
\begin{gathered}
p(i)=n-i+2 \lambda-1 \quad \mu=\frac{n+2 \lambda-1}{2} \\
C_{i i}=\frac{2 \mu^{[p(i)+1] / 2}}{\Gamma((p(i)+1) / 2)} \quad C_{i l}=\sqrt{\frac{\mu}{\pi}}
\end{gathered}
$$

In these equations, $n$ denotes the size of the matrices $\boldsymbol{A}$, i.e. the number of modes retained, and $\Gamma($.$) denotes the$ Gamma function. In fact, it is readily seen that the off-diagonal elements are identically distributed as zero mean Gaussian random variables with standard deviation $1 / \sqrt{2 \mu}$ while the diagonal ones can be simulated as $H_{i i}=\sqrt{\frac{Y_{i i}}{\mu_{i i}}}$ where $Y_{i i}$ are Gamma distributed random variables with parameter $p(i)+1$. The construction of the random matrices $\boldsymbol{H}$ is pictorially summarized in Fig. 1. 


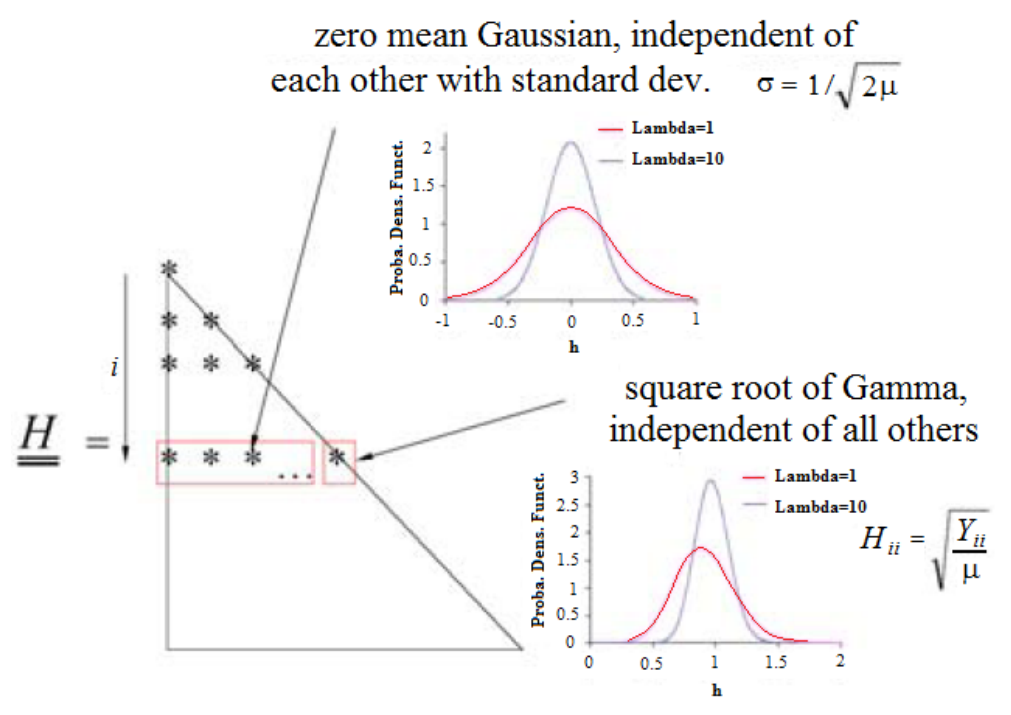

Figure 1. Structure of the random matrices $\boldsymbol{A}$ (figures for $n=8, i=2$, and $\lambda=1$ and 10)

In the above equations, the parameter $\lambda>0$ is the free parameter of the statistical distribution of the random matrices $\boldsymbol{H}$ and $\boldsymbol{A}$ and can be evaluated to meet any given information about their variability. In the ensuing examples, the parameter $\lambda$ will be determined to yield a specified value of the overall measure of uncertainty $\delta$ defined as

$$
\delta^{2}=\frac{1}{n} E\left[\left\|\boldsymbol{H} \boldsymbol{H}^{T}-\boldsymbol{I}\right\|_{F}^{2}\right]=\frac{n+1}{n+2 \lambda-1}
$$

where $\boldsymbol{I}$ is the identity matrix, and \|\|$_{F}$ denotes the Frobenius norm of a matrix. This condition, coupled with Eqs. (1)-(5), provides a complete scheme for the generation of random symmetric positive definite matrices $\boldsymbol{A}$.

It should be recognized that the random matrices $\boldsymbol{A}$ are full irrespectively of any particular structure that the mean matrix $\overline{\boldsymbol{A}}$ may exhibit. This observation implies that the uncertainty it induces is not limited to the structure defined by the mean model or equivalently that model uncertainty is included in the formulation. This property is particularly desirable here given the difficulty in appropriately modeling the boundary conditions. 


\section{Uncertain Clamped Boundary Conditions}

\section{A. Modeling Strategy}

A perfect clamped boundary cannot exhibit any uncertainty as the displacements and slopes are exactly set to zero. The physical problem which is thus modeled is one in which there is flexibility at the boundary and it is that flexibility which is uncertain. The first step in the present effort is then to replace the perfect clamped boundary condition by an "imperfect"/flexible one which is represented by a distribution of springs (both linear and torsional), see Fig. 2. This discussion will be carried out first in the absence of uncertainty in the boundary conditions which will then be introduced in the second step.
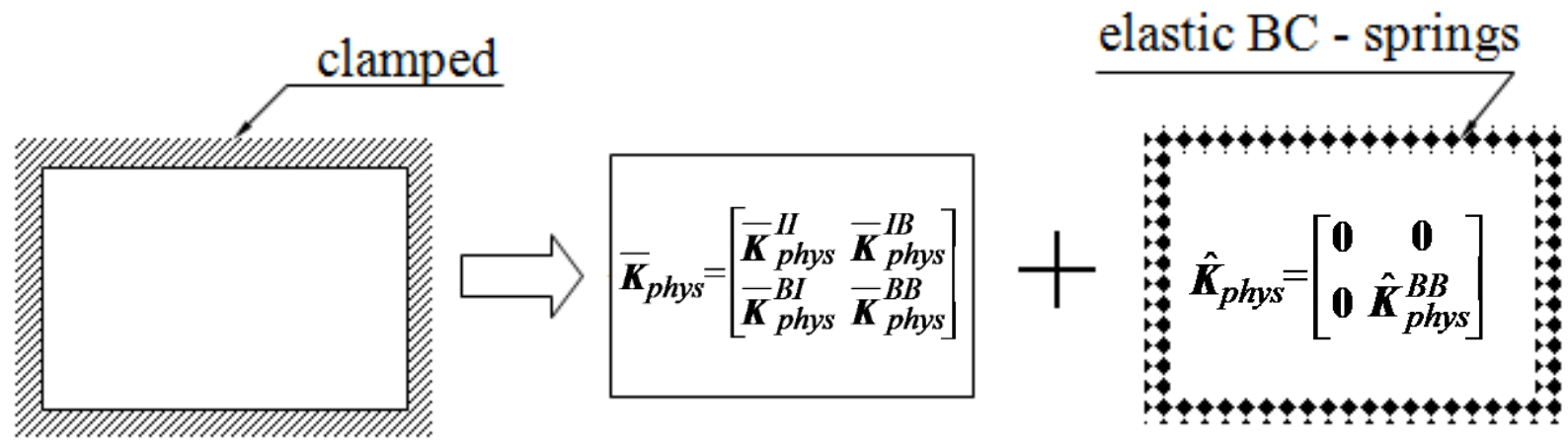

Figure 2. Transformation of the perfect clamped boundary condition into a flexible boundary condition and separation of the domains.

Assuming that the modeling of the structure is accomplished with finite elements, the next step is to proceed with a partitioning of the degrees-of-freedom of the structure with flexible boundary conditions in terms of internal $(I)$ and boundary $(B)$ degrees-of-freedom. Accordingly, the stiffness matrix of the structure may be expressed as

$$
\boldsymbol{K}_{\text {phys }}=\overline{\boldsymbol{K}}_{\text {phys }}+\hat{\boldsymbol{K}}_{\text {phys }}
$$

where, in partitioned form,

$$
\overline{\boldsymbol{K}}_{\text {phys }}=\left[\begin{array}{ll}
\overline{\boldsymbol{K}}_{\text {phys }}^{I I} & \overline{\boldsymbol{K}}_{\text {phys }}^{I B} \\
\overline{\boldsymbol{K}}_{\text {phys }}^{B I} & \overline{\boldsymbol{K}}_{\text {phys }}^{B B}
\end{array}\right]
$$

and

$$
\hat{\boldsymbol{K}}_{\text {phys }}=\left[\begin{array}{cc}
\mathbf{0} & \mathbf{0} \\
\mathbf{0} & \hat{\boldsymbol{K}}_{\text {phys }}^{B B}
\end{array}\right]
$$


Note in this decomposition that $\overline{\boldsymbol{K}}_{\text {phys }}$ is the stiffness matrix of the free-free structure. Assuming that the boundary is massless, one obtains similarly

$$
\boldsymbol{M}_{\text {phys }}=\overline{\boldsymbol{M}}_{\text {phys }}
$$

with

$$
\overline{\boldsymbol{M}}_{\text {phys }}=\left[\begin{array}{ll}
\overline{\boldsymbol{M}}_{\text {phys }}^{I I} & \overline{\boldsymbol{M}}_{\text {phys }}^{I B} \\
\overline{\boldsymbol{M}}_{\text {phys }}^{B I} & \overline{\boldsymbol{M}}_{\text {phys }}^{B B}
\end{array}\right]
$$

A first reduced order model of the structure with flexible boundary conditions can be derived by proceeding with a Craig-Bampton approach (e.g. see [14-17]), i.e. by approximating the internal ( $\boldsymbol{X}_{\text {phys }}^{I}$ ) and boundary $\left(\boldsymbol{X}_{\text {phys }}^{B}\right)$ degrees-of-freedom as

$$
X_{p h y s}^{I}=\Phi q+\boldsymbol{\Xi} Y
$$

and

$$
\boldsymbol{X}_{\text {phys }}^{B}=\boldsymbol{Y}
$$

where $\Phi$ denotes the modal matrix of $p$ selected modes of the clamped structure, i.e. $\Phi=\left[\begin{array}{llll}\phi_{1} & \phi_{2} & \cdots & \phi_{p}\end{array}\right]$, where

$$
\overline{\boldsymbol{K}}_{\text {phys }}^{I I} \boldsymbol{\phi}_{j}=\omega_{C, j}^{2} \overline{\boldsymbol{M}}_{\text {phys }}^{I I} \boldsymbol{\phi}_{j}
$$

Further, in Eq. (11), the symbol $\boldsymbol{\Xi}$ denotes the matrix of constraint modes

$$
\boldsymbol{\Xi}=-\left(\overline{\boldsymbol{K}}_{\text {phys }}^{I I}\right)^{-1} \overline{\boldsymbol{K}}_{\text {phys }}^{I B}
$$

Finally, the vector $\boldsymbol{q}$ denotes the generalized coordinates of the modes of the clamped structure.

The reduction of variables, from $\left(\boldsymbol{X}_{\text {phys }}^{I}, \boldsymbol{X}_{\text {phys }}^{B}\right)$ to $(\boldsymbol{q}, \boldsymbol{Y})$, is accompanied by the matrix

$$
\boldsymbol{T}_{1}=\left[\begin{array}{cc}
\Phi & \Xi \\
\mathbf{0} & \boldsymbol{I}
\end{array}\right]
$$

where $\boldsymbol{I}$ denotes the identity matrix of appropriate dimensions. Thus, the stiffness and mass matrices of the free-free structure associated with the variables $(\boldsymbol{q}, \boldsymbol{Y})$ are 


$$
\boldsymbol{K}_{C B}=\boldsymbol{T}_{1}^{T} \overline{\boldsymbol{K}}_{\text {phys }} \boldsymbol{T}_{1}=\left[\begin{array}{ll}
\boldsymbol{K}_{C B}^{q q} & \boldsymbol{K}_{C B}^{q Y} \\
\boldsymbol{K}_{C B}^{Y q} & \boldsymbol{K}_{C B}^{Y Y}
\end{array}\right]
$$

and

$$
\boldsymbol{M}_{C B}=\boldsymbol{T}_{1}^{T} \overline{\boldsymbol{M}}_{\text {phys }} \boldsymbol{T}_{1}=\left[\begin{array}{ll}
\boldsymbol{M}_{C B}^{q q} & \boldsymbol{M}_{C B}^{Y Y} \\
\boldsymbol{M}_{C B}^{Y q} & \boldsymbol{M}_{C B}^{Y Y}
\end{array}\right]
$$

Since the reduced order model is built on the modal matrix $\boldsymbol{\Phi}$, the matrices $\boldsymbol{K}_{C B}^{q q}$ and $\boldsymbol{M}_{C B}^{q q}$ are diagonal, and more specifically with nonzero elements equal respectively to the natural frequencies and to 1 if the modes $\phi_{j}$ have been normalized with respect to the mass matrix $\overline{\boldsymbol{M}}_{\text {phys }}^{I I}$.

The reduced order model of Eq. (11) and (12) is in fact "mixed" as it contains both modal coordinates (for the internal degrees-of-freedom) and physical coordinates (for the boundary degrees-of-freedom). A "fully" reduced order model can be developed by expressing the physical boundary degrees-of-freedom as

$$
\boldsymbol{Y}=\Psi \boldsymbol{u}
$$

where $\Psi=\left[\begin{array}{llll}\Psi_{1} & \psi_{2} & \cdots & \Psi_{r}\end{array}\right]$ and the vectors $\Psi_{j}$ are an appropriate basis for the representation of the physical boundary degrees-of-freedom, for instance the eigenvectors corresponding to $\boldsymbol{K}_{C B}^{Y Y}$ and $\boldsymbol{M}_{C B}^{Y Y}$. That is, satisfying

$$
\boldsymbol{K}_{C B}^{Y Y} \boldsymbol{\psi}_{j}=\lambda_{j} \boldsymbol{M}_{C B}^{Y Y} \boldsymbol{\psi}_{j}
$$

This second reduction of degrees-of-freedom is accompanied by the matrix

$$
\boldsymbol{T}_{2}=\left[\begin{array}{ll}
\boldsymbol{I} & \mathbf{0} \\
\mathbf{0} & \Psi
\end{array}\right]
$$

and thus, the stiffness and mass matrices of the free-free structure associated with the variables $(\boldsymbol{q}, \boldsymbol{u})$ are

$$
\overline{\boldsymbol{K}}_{R O M}=\boldsymbol{T}_{2}^{T} \boldsymbol{K}_{C B} \boldsymbol{T}_{2}=\left[\begin{array}{ll}
\overline{\boldsymbol{K}}_{R O M}^{q q} & \overline{\boldsymbol{K}}_{R O M}^{q u} \\
\overline{\boldsymbol{K}}_{R O M}^{u q} & \overline{\boldsymbol{K}}_{R O M}^{u u}
\end{array}\right]
$$

and

$$
\overline{\boldsymbol{M}}_{R O M}=\boldsymbol{T}_{2}^{T} \boldsymbol{M}_{C B} \boldsymbol{T}_{2}=\left[\begin{array}{ll}
\overline{\boldsymbol{M}}_{R O M}^{q q} & \overline{\boldsymbol{M}}_{R O M}^{q u} \\
\overline{\boldsymbol{M}}_{R O M}^{u q} & \overline{\boldsymbol{M}}_{R O M}^{u u}
\end{array}\right]
$$


The above discussion focused solely on the free-free structure but the consideration of its flexible boundary counterpart is accomplished simply through the addition of the finite boundary stiffness matrix $\hat{\boldsymbol{K}}_{p h y s}$, see Eq. (7) and (8). That is, $\boldsymbol{K}_{R O M}=\overline{\boldsymbol{K}}_{R O M}+\hat{\boldsymbol{K}}_{R O M}$ where

$$
\hat{\boldsymbol{K}}_{R O M}=\boldsymbol{T}_{2}^{T} \boldsymbol{T}_{1}^{T} \hat{\boldsymbol{K}}_{p h y s} \boldsymbol{T}_{1} \boldsymbol{T}_{2}=\left[\begin{array}{cc}
\mathbf{0} & \mathbf{0} \\
\mathbf{0} & \hat{\boldsymbol{K}}_{R O M}^{u u}
\end{array}\right]
$$

In practical situations, the matrix $\hat{\boldsymbol{K}}_{\text {phys }}$ is generally not known which in fact is why a perfect clamped boundary condition is often introduced. The next level of complexity, which will be adopted here, is to relate $\hat{\boldsymbol{K}}_{\text {phys }}$ to the boundary-boundary partition of the stiffness matrix of the free-free structure. This relation is most conveniently achieved directly in the reduced order model variables, i.e. by assuming

$$
\hat{\boldsymbol{K}}_{R O M}^{u u}=k \Psi^{T} \overline{\boldsymbol{K}}_{\text {phys }}^{B B} \Psi
$$

in which $k$ is a scalar that constitutes a parameter of the boundary condition modeling.

Combining the preceding results, it is found that the overall ROM stiffness matrix is

$$
\boldsymbol{K}_{R O M}=\overline{\boldsymbol{K}}_{R O M}+\hat{\boldsymbol{K}}_{R O M}=\left[\begin{array}{cc}
\overline{\boldsymbol{K}}_{R O M}^{q q} & \overline{\boldsymbol{K}}_{R O M}^{q u} \\
\overline{\boldsymbol{K}}_{R O M}^{u q} & \overline{\boldsymbol{K}}_{R O M}^{u u}+\hat{\boldsymbol{K}}_{R O M}^{u u}
\end{array}\right]
$$

The determination of the natural frequencies $\omega_{f, j}$ and mode shapes $\varphi_{j}$ of the flexible boundary structure is achieved by solving the eigenvalue problem

$$
\boldsymbol{K}_{R O M} \boldsymbol{\varphi}_{R O M, j}=\omega_{f, j}^{2} \overline{\boldsymbol{M}}_{R O M} \boldsymbol{\varphi}_{R O M, j} \quad \text { then } \quad \boldsymbol{\varphi}_{j}=\boldsymbol{T}_{1} \boldsymbol{T}_{2} \boldsymbol{\varphi}_{R O M, j}
$$

The consideration of uncertainty of the free-free structure is easily performed from Eq. (25) through the nonparametric approach as in [11], see also [1]. Specifically, if the free-free structure is uncertain, a random reduced order stiffness matrix $\breve{\boldsymbol{K}}_{R O M}$ can be obtained as $\breve{\boldsymbol{K}}_{R O M}=\overline{\boldsymbol{L}}_{R O M} \boldsymbol{H} \boldsymbol{H}^{T} \overline{\boldsymbol{L}}_{R O M}^{T}+\hat{\boldsymbol{K}}_{R O M}$ where $\overline{\boldsymbol{L}}_{R O M}$ is the Cholesky decomposition of $\overline{\boldsymbol{K}}_{R O M}$, i.e. the lower triangular matrix satisfying the equation $\overline{\boldsymbol{K}}_{R O M}=\overline{\boldsymbol{L}}_{R O M} \overline{\boldsymbol{L}}_{R O M}^{T}$. Further $\boldsymbol{H}$ denotes the random matrix of Eqs (2)-(5), see also Fig. 1. Uncertainty in boundary conditions alone is introduced similarly by replacing the mean model matrix $\hat{\boldsymbol{K}}_{R O M}^{u u}$ by $\breve{\boldsymbol{K}}_{R O M}^{u u}=k \overline{\boldsymbol{L}}_{R O M}^{u u} \boldsymbol{H}^{u u}\left(\boldsymbol{H}^{u u}\right)^{T}\left(\overline{\boldsymbol{L}}_{R O M}^{u u}\right)^{T}$ 
where $\overline{\boldsymbol{L}}_{R O M}^{u u}$ is the Cholesky decomposition of $\hat{\boldsymbol{K}}_{R O M}^{u u}=\overline{\boldsymbol{L}}_{R O M}^{u u}\left(\overline{\boldsymbol{L}}_{R O M}^{u u}\right)^{T}$ and $\boldsymbol{H}^{u u}$ is another random matrix also defined by Eqs (2)-(5), see also Fig. 1. Note that the matrices $\boldsymbol{H}$ and $\boldsymbol{H}^{u u}$ model different sources of uncertainties, i.e. on the free-free structure and on its boundary conditions, respectively, and thus may or may not be correlated. This issue is not considered further in this paper which focuses solely on lesser studied uncertainty in the boundary conditions.

It should finally be recognized that the use of the nonparametric approach permits to account for model uncertainty as the random matrices $\breve{\boldsymbol{K}}_{R O M}^{u u}$ are full matrices, i.e. they do not conform to the specific form chosen for the mean model. This is in contrast with prior investigations in which the values of the stiffnesses used in the mean model are randomized. In such investigations, only parameter uncertainty can be induced, the form of the stiffness matrix $\breve{\boldsymbol{K}}_{R O M}^{u u}$ remaining the same as that of $\hat{\boldsymbol{K}}_{R O M}^{u u}$.

\section{B. Examples of Application}

To demonstrate the process discussed above and clarify the effects of the parameters $k$ and $\delta$, an aluminum clamped plate of dimension $0.3556 \mathrm{~m} \times 0.254 \mathrm{~m} \times 0.001 \mathrm{~m}$ was considered. The material properties of aluminum were selected as $E=70,000 \mathrm{MPa}, v=0.30, \rho=2700 \mathrm{~kg} / \mathrm{m}^{3}$. A first set of computations was carried out without uncertainty to analyze the mean model and in particular the effects of $k$ on the natural frequencies and mode shapes. The results of this analysis are presented in Fig. 3 for the first seven natural frequencies and mode shapes. The ordinate of Fig. 3(a) is the ratio of the natural frequencies to their $k=\propto$ counterparts while those of Fig. 3(b) are the ratios of the norm of the difference between each mode and its $k=\propto$ counterpart divided by the norm of the latter. As expected, both natural frequencies and mode shapes converge monotonically as $k \rightarrow \propto$ to those of the perfectly clamped plate and with higher order modes converging faster as they are less sensitive to the boundary modeling. In all ensuing discussions, the value of $k=0.75$ was selected.

It was next desired to assess the convergence of the model prediction with increasing number of boundary modes $\Psi_{j}$ which is guaranteed since these modes span the space of the boundary deflections. Thus, the inclusion of all 240 boundary modes will recover the original Craig-Bampton model of Eqs (16) and (17) only expressed in terms of different boundary coordinates. A typical convergence plot of the natural frequencies to their Craig-Bampton 
counterparts is shown in Fig. 4 which indicates that an accurate reduced order model is accomplished with only a small number of boundary modes. The small magnitudes of the ordinates in Fig. 4 suggest that the boundary flexibility has only a small effect on the modes shapes. This expectation is confirmed in Fig. 5 which shows the first

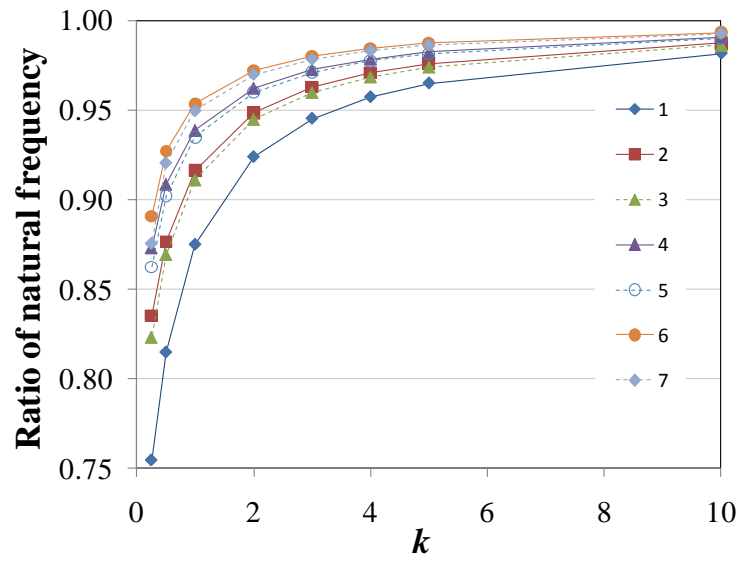

(a)

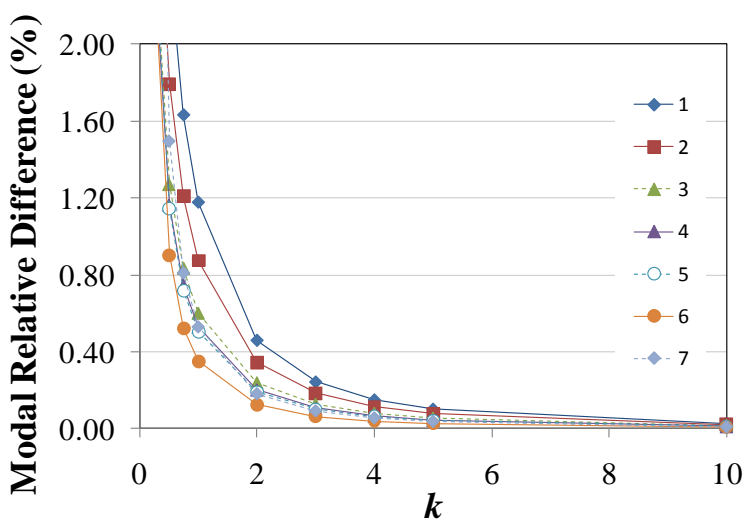

(b)

Figure 3. Convergence of the first seven (a) natural frequencies and (b) mode shapes as a function of $k$ to their $k=\propto$ counterparts, all boundary modes included.

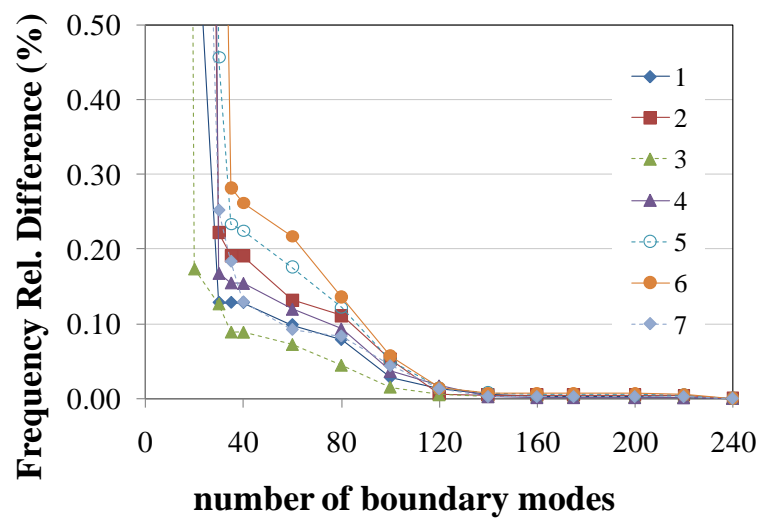

(a)

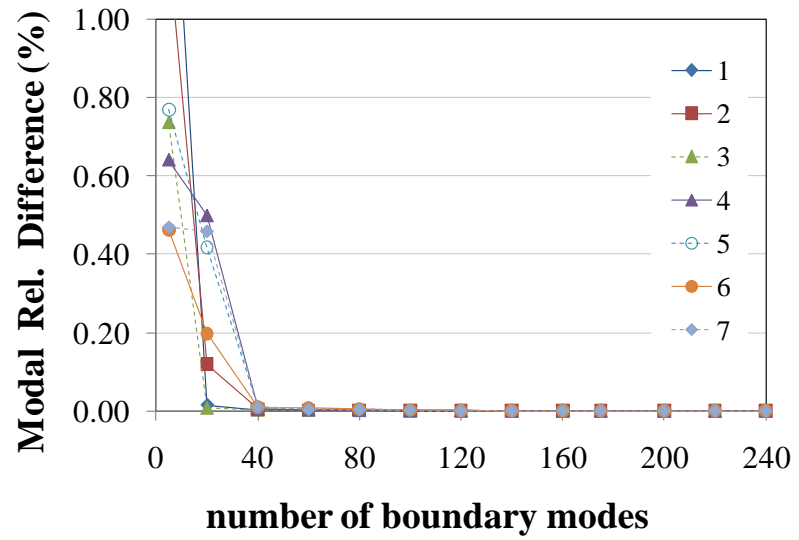

(b)

Figure 4. Convergence of the first seven (a) natural frequencies and (b) mode shapes with increasing number of boundary modes, $k=0.75$.

4 modes of the flexible boundary condition plate with $k=0.75$ which are very similar to those obtained for a perfectly rigid boundary, i.e. for $k=\propto$, the most noticeable differences being slight displacements and rotations at the boundary.

To obtain a better perspective of the differences between the flexible and rigid boundary mode shapes, consider the vectors

$$
\Delta \varphi_{j}=\varphi_{j}-\left(\varphi_{j}^{(\infty) T} M_{p h y s} \varphi_{j}\right) \varphi_{j}^{(\infty)}
$$


where $\varphi_{j}^{(\infty)}$ denotes the mass normalized mode shapes of the structure with rigid boundary. Then, $\Delta \varphi_{j}$ represents the remainder of the projection, or vector rejection, of the flexible boundary mode $\varphi_{j}$ on its rigid counterpart $\varphi_{j}^{(\infty)}$ and thus can be used to assess the effects of the boundary flexibility. The plots of $\Delta \varphi_{j}$ for the first four modes, see

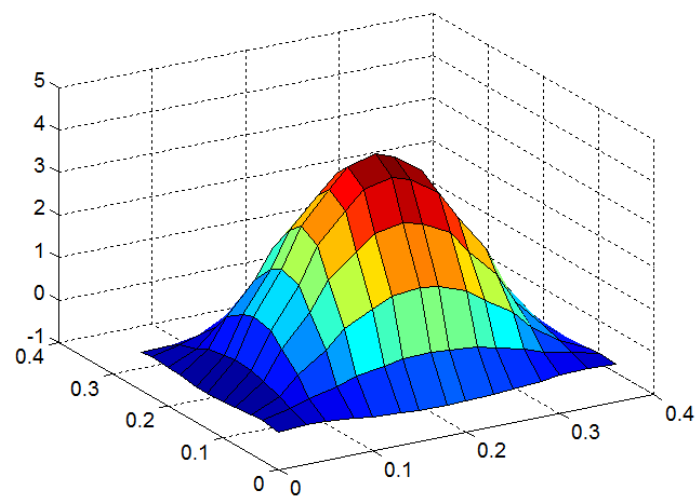

(a)

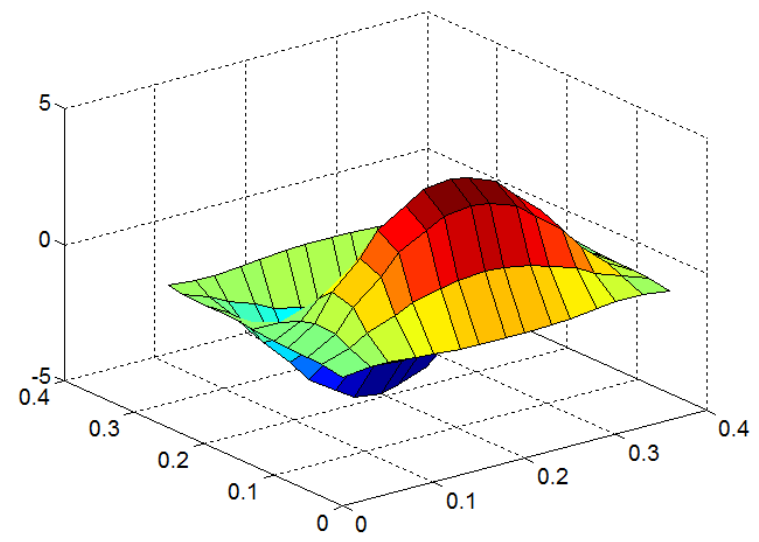

(c)

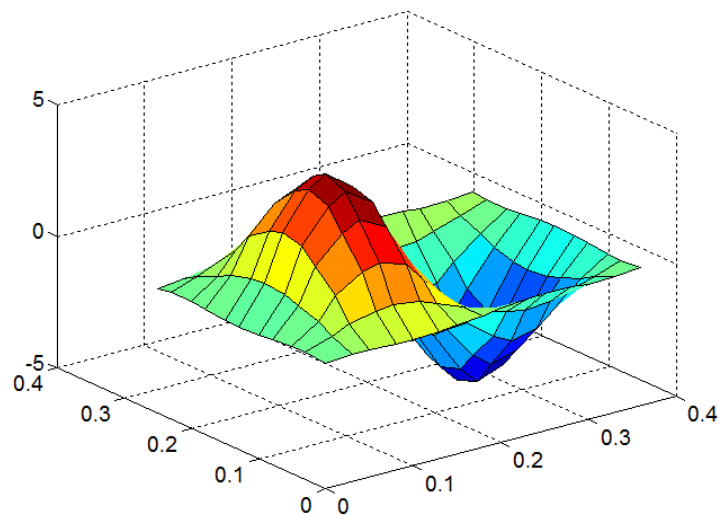

(b)

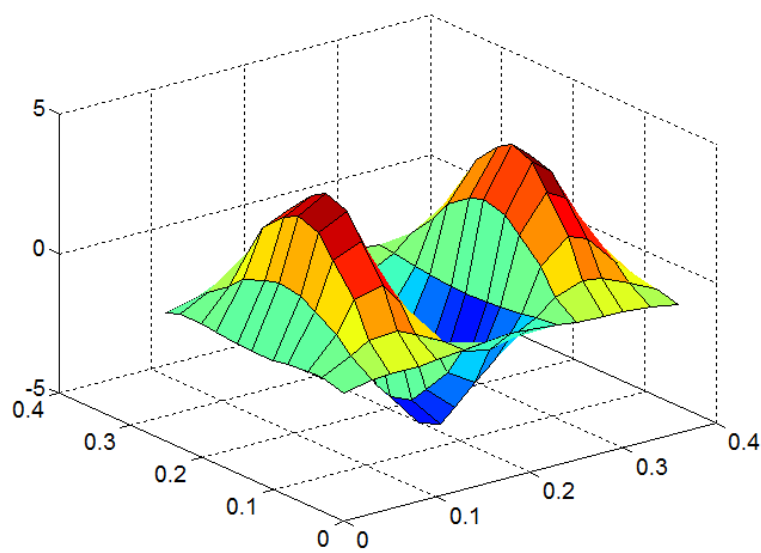

(d)

Figure 5. First four modes of the flexible boundary plate, $k=0.75,120$ boundary modes, 10 clamped modes. (a),(b),(c),(d) modes 1, 2, 3, and 4.

Fig. 6, confirm the above preliminary observation that the boundary flexibility affects the modes of the plate near the boundary but, to some extent, also away from it.

Uncertainty was introduced next in the model using the nonparametric methodology, Eqs (1)-(6), and an ensemble of matrices $\breve{\boldsymbol{K}}_{R O M}^{u u}$ were generated for $k=0.75$ but with different levels of uncertainty, i.e. different values of the parameter $\delta$, see Eq. (6). First assessed were the effects of the number of boundary modes included in the model on the mean and standard deviations of the natural frequencies and it was found that the mean values converged must faster than the corresponding standard deviations as the number of boundary modes retained increased. The analysis was first carried out for a constant value of the parameter $\delta$, see Fig. 7(a). Surprisingly, it was observed that the 
standard deviations of the natural frequencies first increased with increasing number of modes then decreased. The first phase, increase with increasing number of modes, is expected since each new mode induces further uncertainty in the model. Of course, the effects of additional modes decrease owing to the convergence of the natural frequencies with the number of boundary modes retained, see Fig. 4(a). Thus, one would expect the increase in standard deviations of the frequencies to flatten out leading to a somewhat monotonic convergence. This is however not what is observed, see Fig. 7(a): the standard deviations exhibit a peak at 25 or so boundary modes and then steadily decrease thereafter.

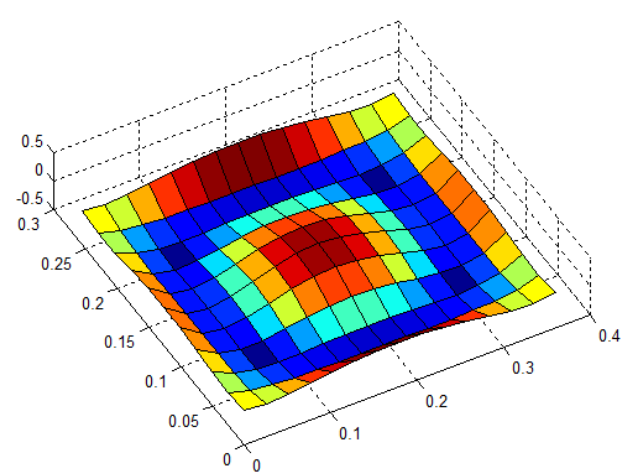

(a)

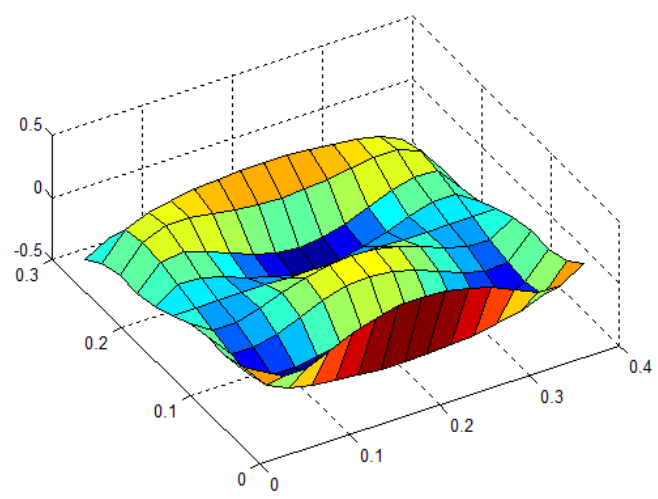

(c)

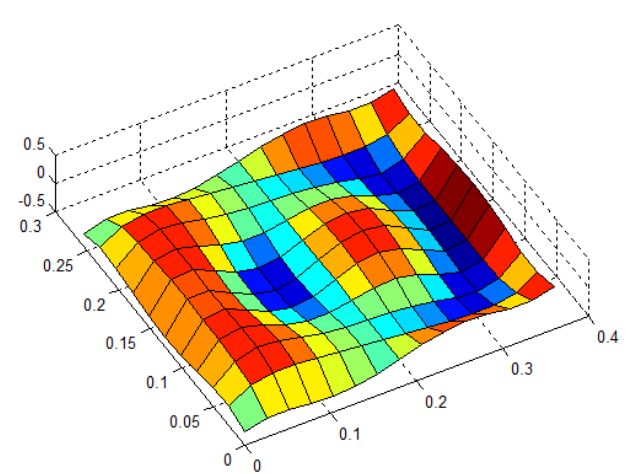

(b)

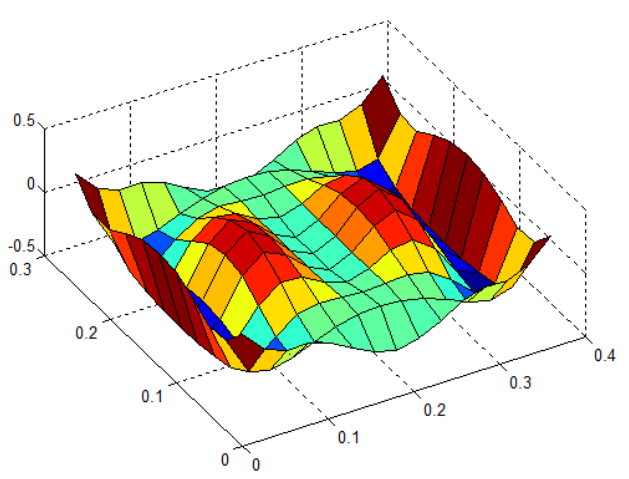

(d)

Figure 6. Remainder $\Delta \varphi_{j}$ of the projection, or vector rejection, of the modes of Fig. 5 on their rigid boundary counterparts. (a),(b),(c),(d) modes 1, 2, 3, and 4.

In clarifying this trend, it was observed from Eqs (4) and (6) that the parameter $\mu$ increases as the number of modes retained increases (it is the parameter $n$ in these equations). Thus, the standard deviation of each component of $\boldsymbol{H}^{u u}$ decreases and accordingly there is less uncertainty associated with each given boundary mode. Combining this observation with the lessened effects of higher order boundary modes, see Fig. 4(a), leads indeed to the decreasing behavior of Fig. 7(a). The expected, monotonic convergence of the standard deviations of the natural 
frequencies with the number of boundary modes is obtained by keeping $\lambda$ constant, for which $\mu$ is also close to constant if $\lambda \gg>n$ as is the case here, see Fig. 7(b). Note again the rapid convergence with only a few boundary modes confirming the observation drawn in connection with the mean model.

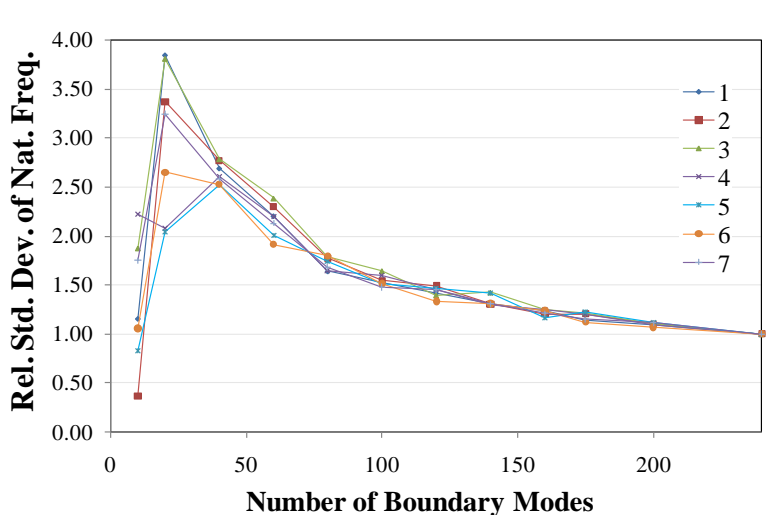

(a)

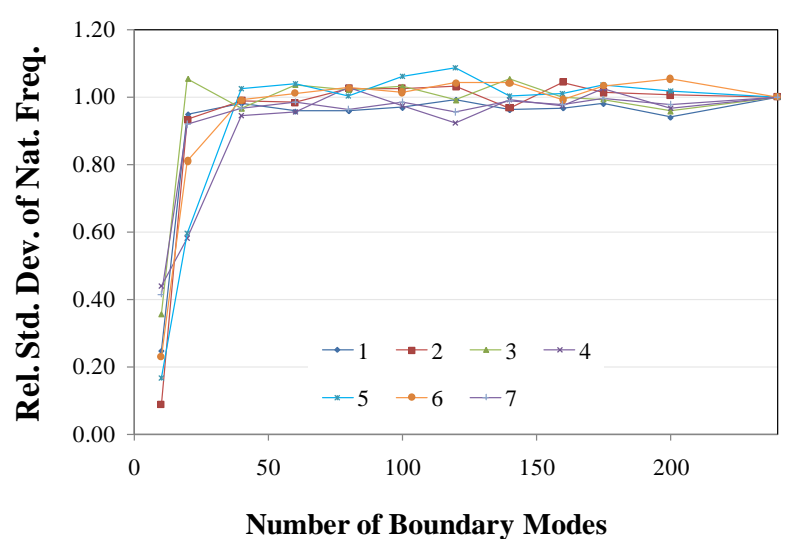

(b)

Figure 7. Standard deviation of the 7 lowest natural frequencies divided by the asymptotic value (in \%), as a function of the number of boundary modes, $k=0.75$, (a) $\delta=0.1$ constant (b) $\lambda=4950$ constant.

In summary, for a fixed number of boundary modes, the parameters $\delta$ and $\lambda$ are fully equivalent to measure the level of uncertainty with the former one preferred because of its direct physical interpretation of Eq. (6). However, when varying the number of boundary modes, it is suggested that $\lambda$ be kept constant as justified above.

The model of uncertain boundary conditions developed in the previous section is a 2 parameter model as it involves the coefficient $k$ of Eq. (24) and the uncertainty measure $\delta$ of Eq. (6). In fact, shown in Fig. 8 are the coefficients of variations of the first two natural frequencies, as functions of $k$ and $\delta$. These plots do exhibit expected behaviors where the coefficients of variations both grow as a function of the uncertainty measure for all values of $k$. Second, these coefficients of variations are also monotonically decreasing function of $k$ as might be expected since the limit $k \rightarrow \infty$ should recover the perfectly clamped plate for which the natural frequencies do not exhibit any variability. 


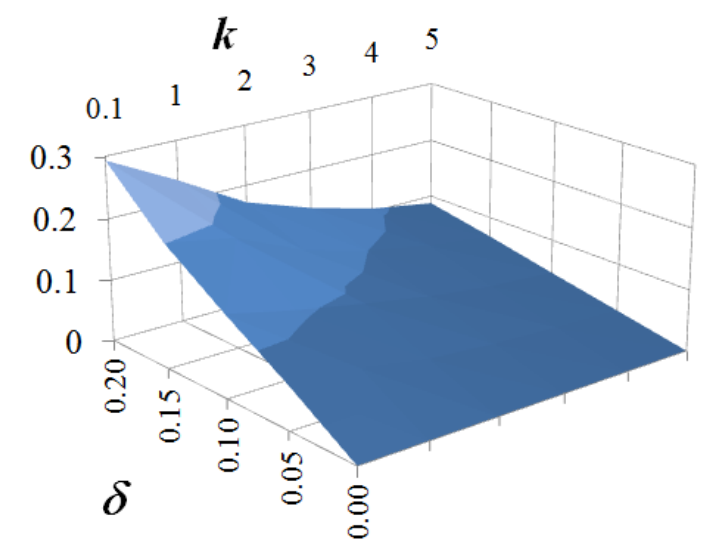

(a)

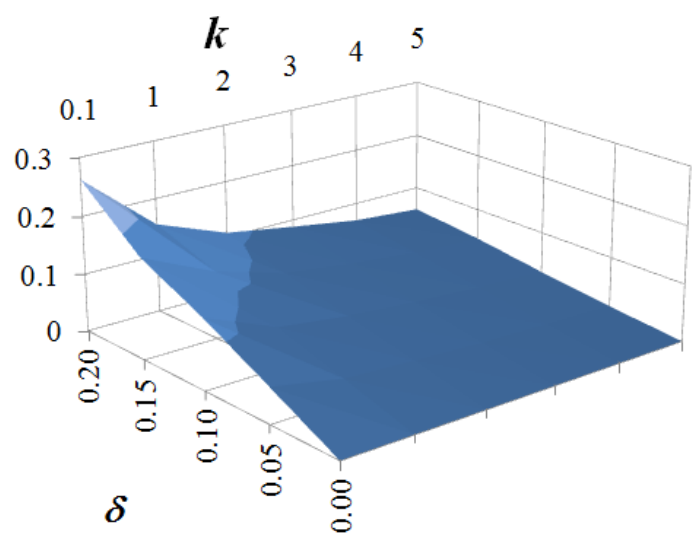

(b)

Figure 8. Coefficients of variation (in \%) of the first two natural frequency vs. $k$ and $\delta, 120$ boundary modes, 10 clamped modes. (a) first mode, (b) second mode.

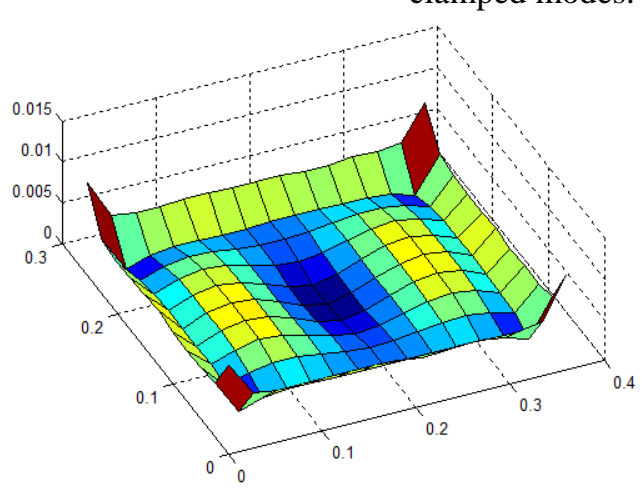

(a)

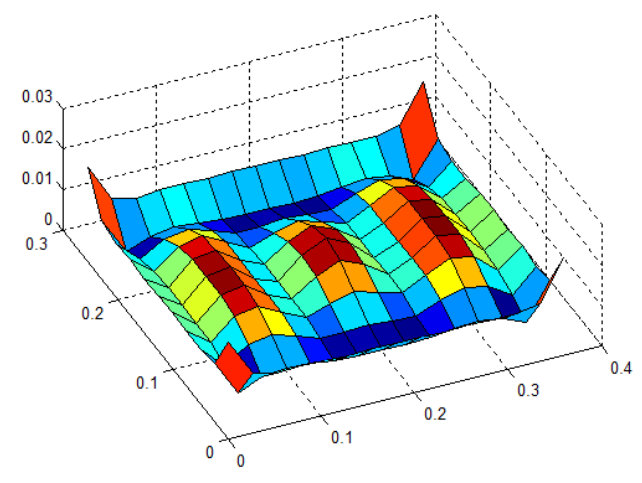

(c)

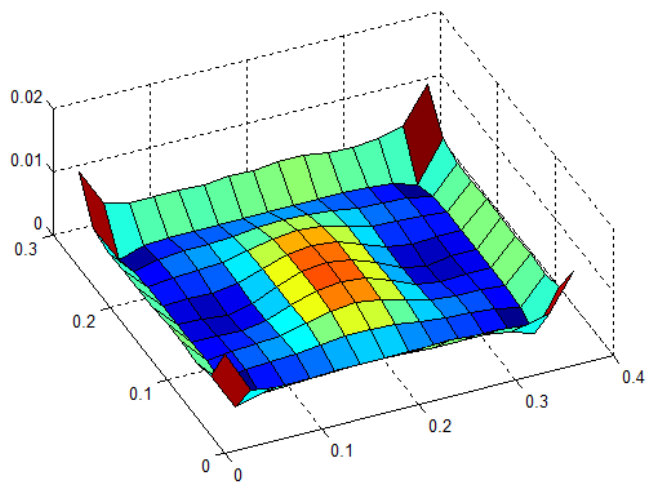

(b)

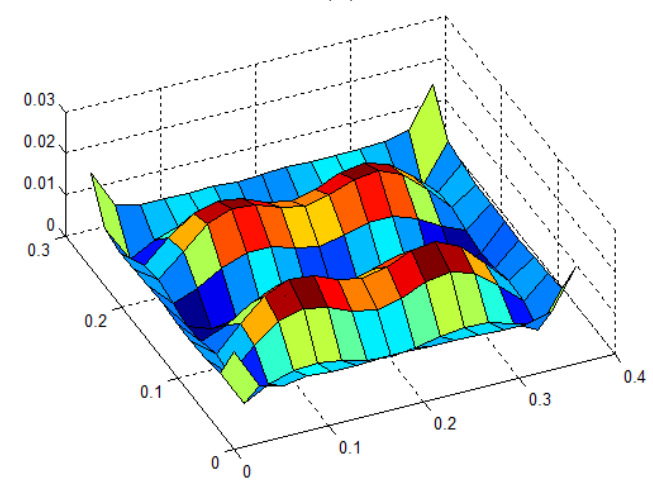

(d)

Figure 9. Standard deviation of modal values, first 4 modes, $k=0.75,120$ boundary modes, 10 clamped modes, $\delta=0.1,(\mathrm{a}),(\mathrm{b}),(\mathrm{c}),(\mathrm{d})$ modes $1,2,3$, and 4.

The effects that uncertainty has on the mode shapes was also investigated, e.g. see Fig. 9 for the standard deviation of the first 4 modes. Since the uncertainty affects only the boundary flexibility, it may be expected that the variability of the mode shapes (e.g. the standard deviations of Fig. 9) will be large where this flexibility plays an important role, i.e. in zones of large values of the discrepancies of Eq. (27), see Fig. 6. Notwithstanding differences 
between Figs 6 and 9, they both exhibit large values in the neighborhood of the boundary identifying this zone as one in which the effects of flexibility and uncertainty are important.

To complete the modeling process, it remains to address the determination/estimation of the two parameters of the boundary conditions uncertainty model, i.e. $k$ and $\delta$, from experimental measurements. Specific identification techniques of these parameters would depend on the information that is measured, i.e. type, number, and location of sensors, as well as on the number of specimen of the structure which are tested. Before such detailed techniques are devised, however, it is necessary to demonstrate that these parameters can indeed be identified and to assess from what information they could be extracted. These latter issues are addressed below assuming a broad availability of response data for a large number of specimen while the formulation of specific identification techniques are the focus of a future experimental validation.

Given the above observation that flexibility $(k)$ and uncertainty $(\delta)$ both affect the mode shapes strongly near the boundary, it is proposed here to focus on metrics that relate to the motions at/near the boundary. An additional benefit of using such metrics is that they are expected to be only weakly dependent on uncertainty in the structure away from the boundary (e.g. on the Young's modulus) not considered here. More specifically then, consider the boundary condition "energy" term $E_{B C}$ defined as

$$
E_{B C}=\left(\boldsymbol{X}_{\text {phys }}^{B}\right)^{T} \boldsymbol{A}_{B C} \boldsymbol{X}_{\text {phys }}^{B}
$$

where $\boldsymbol{A}_{B C}$ is a specified positive definite matrix and $\boldsymbol{X}_{\text {phys }}^{B}$ is a particular response of the boundary. It is then desired to assess the existence of strong correlation between $E_{B C}$ and the parameters $k$ and $\delta$. Shown in Fig. 10 are the mean and coefficient of variation $E_{B C}$ with $A_{B C}$ chosen as a diagonal matrix with elements equal to 1 on translations and 10 on rotations and $\boldsymbol{X}_{\text {phys }}^{B}$ the response at the boundary of the first mode $\boldsymbol{\varphi}_{1}$. Clearly the mean of $E_{B C}$ depends almost solely on $k$, from which it can thus be extracted. Then, the standard deviation of $E_{B C}$ can be used to estimate $\delta$. Thus, the first two moments of the quantity $E_{B C}$ provides straightforward estimates of $k$ and $\delta$. 


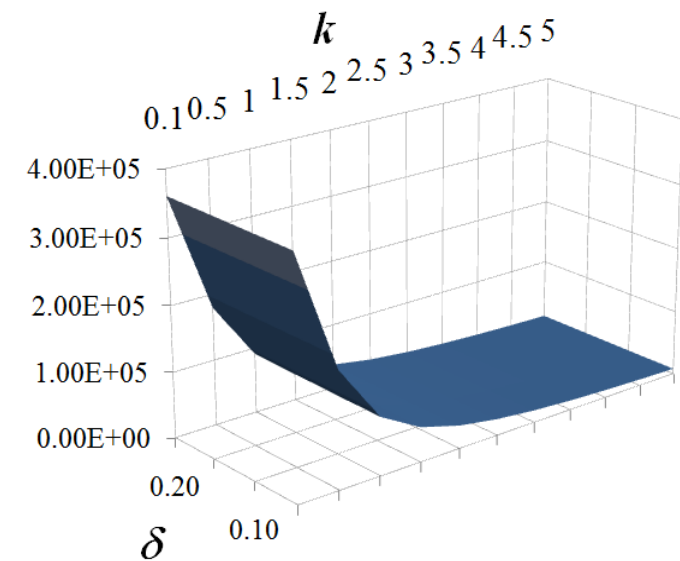

(a)

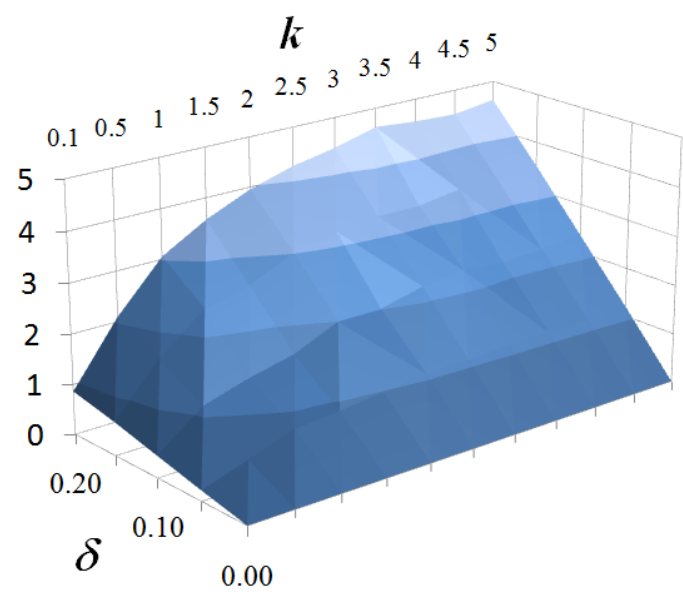

(b)

Figure 10. Mean and coefficient of variation (in \%) of $E_{B C}$, first mode deformations, vs. $k$ and $\delta, 120$ boundary modes, 10 clamped modes.

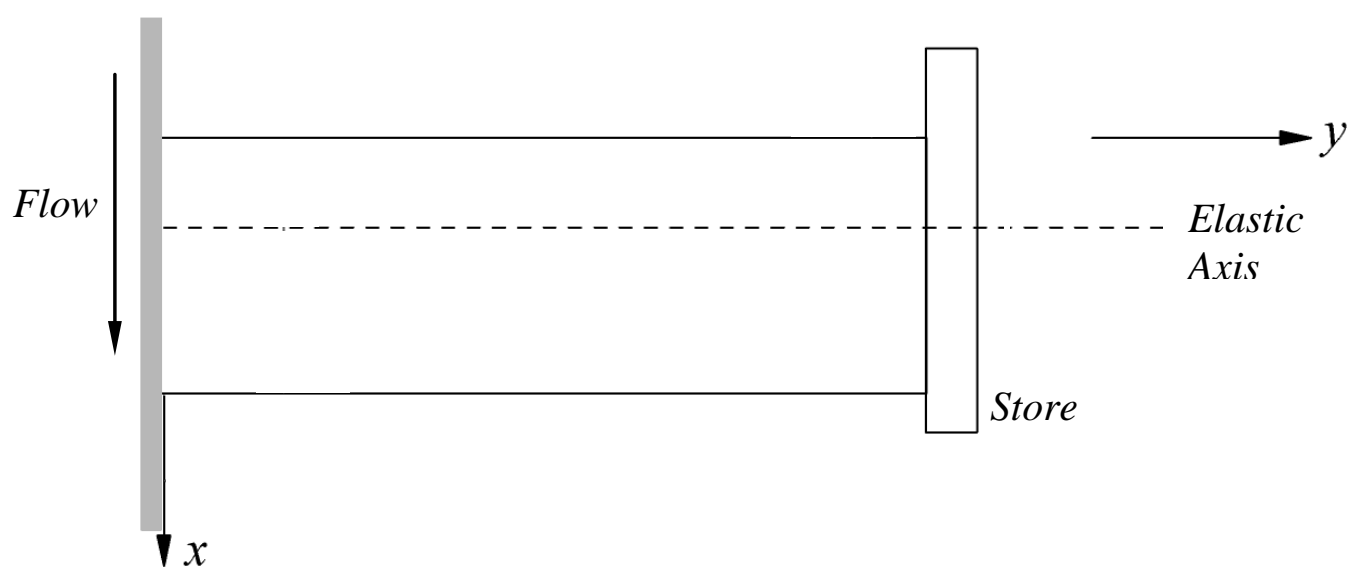

(a)

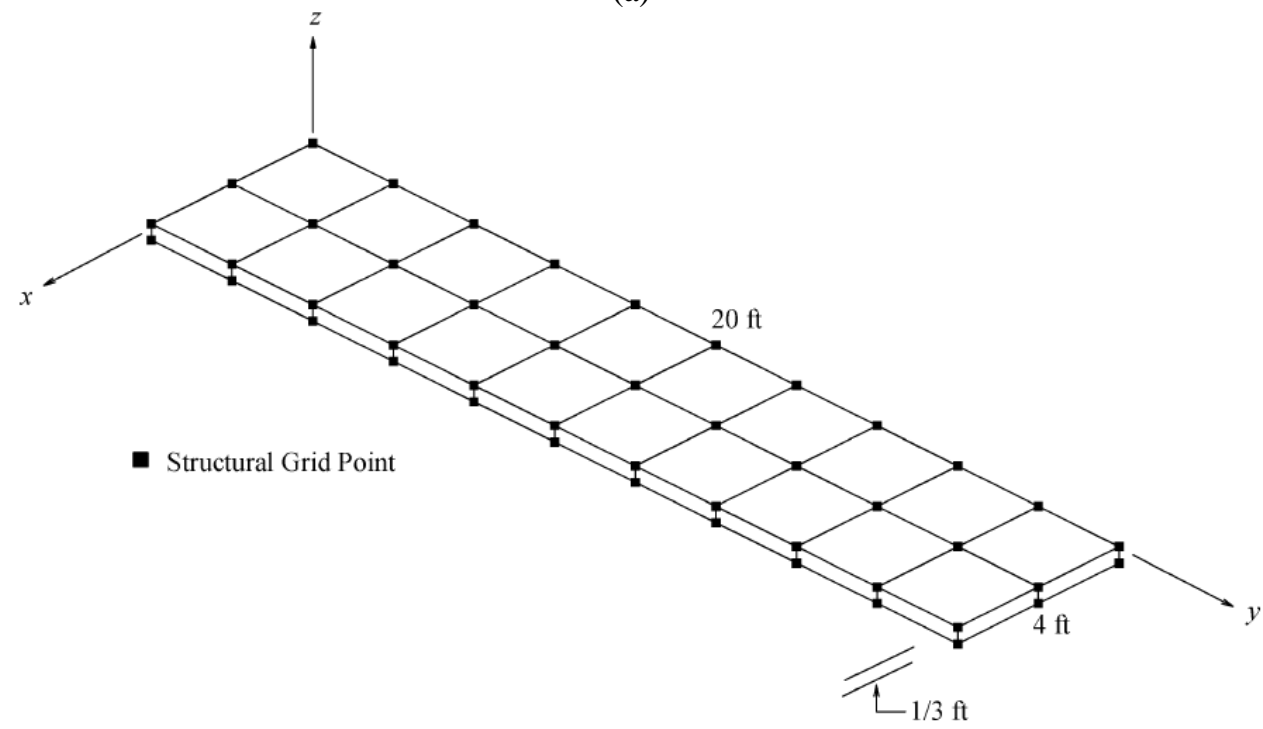

(b)

Figure 11. The Goland+ wing model. (a) Planform geometry, (b) finite element model. (from [19]). 
The analysis of the effects of uncertainty in the boundary conditions can extend further than the natural frequencies and mode shapes of the structure, e.g. to the flutter boundary. To exemplify this application, the Goland+ wing [18-20], see Fig. 11, was considered. It is a flat rectangular wing cantilevered at one end and carrying out a rigid store at the other end modeled as a box structure with skin panels, ribs, and spars (see [19] for detailed geometry and properties). Its first natural frequencies are shown in Table 1 as predicted by Nastran.

\begin{tabular}{|c|c|c|c|}
\hline Mode \# & Nat. Freq. (Hz) & Mode \# & Nat. Freq. (Hz) \\
\hline 1 & 1.690 & 6 & 16.260 \\
\hline 2 & 3.051 & 7 & 22.845 \\
\hline 3 & 9.172 & 8 & 26.318 \\
\hline 4 & 10.834 & 9 & 29.183 \\
\hline 5 & 11.258 & & \\
\hline
\end{tabular}

Table 1. Natural frequencies of the mean Goland+ wing.

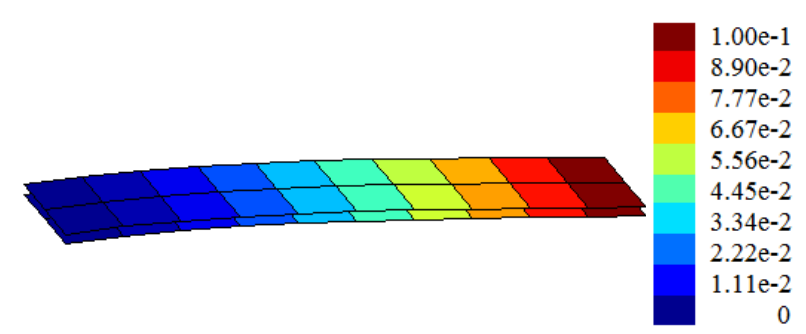

(a)

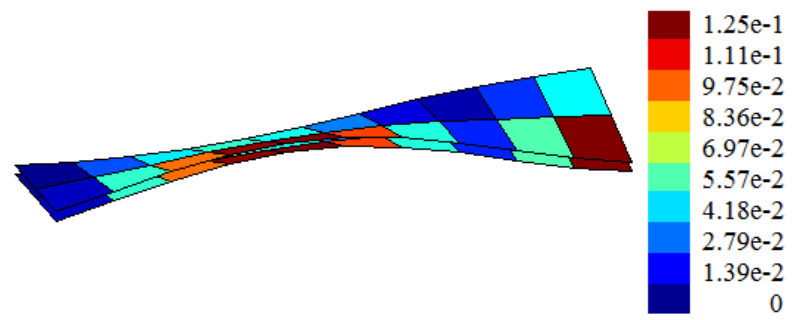

(c)

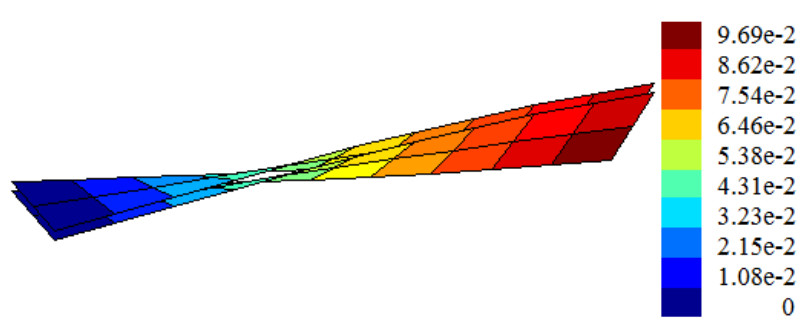

(b)

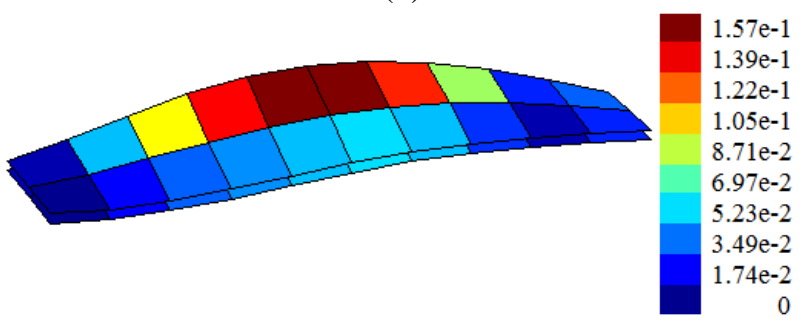

(d)

Figure 12. Mean of modal values of the Goland+ wing, first 4 modes, $k=20,8$ boundary modes, 12 clamped modes, $\delta=0.6,(a),(b),(c),(d)$ modes $1,2,3$, and 4.

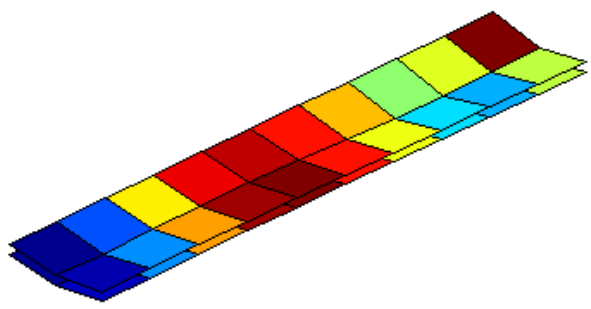

(a)

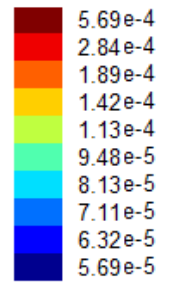

$5.69 \mathrm{e}-5$

American Institute of Aeronautics and Astronautics 


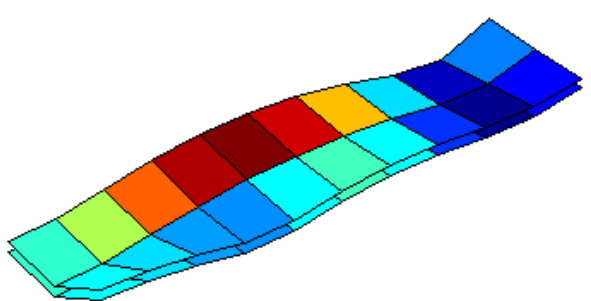

(c)

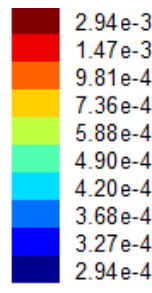

$94 \mathrm{e}-4$

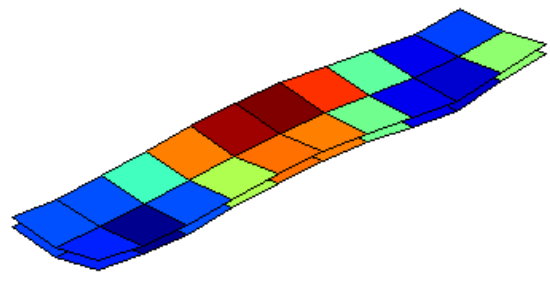

(d)

Figure 13. Standard deviation of modal values of the Goland+ wing, first 4 modes, $k=20,8$ boundary modes, 12 clamped modes, $\delta=0.6$, (a),(b),(c),(d) modes 1, 2, 3, and 4 .

An analysis similar to the one carried out with the clamped plate example was first performed and the parameter $k$ was selected as $k=20$. Further, 12 cantilevered modes and all 8 boundary modes were considered in the analysis. These parameter values led to first and second natural frequencies without uncertainty equal to $98.8 \%$ and $99.4 \%$ of their fully clamped counterparts. Further, the value $\delta=0.6$ was chosen; it leads to coefficients of variation of the first and second natural frequencies of $0.28 \%$ and $0.14 \%$, respectively, see also Figs 12 and 13 for the mean and standard deviation of the first four modes of the flexible support wing.

The flutter boundary of the fully clamped wing model was first determined for a Mach number $M_{\infty}=0.7$ using the ZONA Technology code ZAERO (see $[15,16]$ for related investigations). It was found that flutter occurs at $752.87 \mathrm{ft} / \mathrm{s}$ with a frequency of $1.966 \mathrm{~Hz}$. Next, an ensemble of 300 uncertain wings (with flexible boundary conditions) were simulated and their flutter boundary was determined using ZAERO for $M_{\infty}=0.7$ with the 20 mode model based on the mean wing with flexible boundary conditions, i.e. the 12 cantilevered modes and the 8 boundary modes. Then, shown in Fig. 14 are the first and second natural frequencies of these wings (Fig. 14(a)) and their corresponding matched point flutter boundaries (Fig. (14b)).

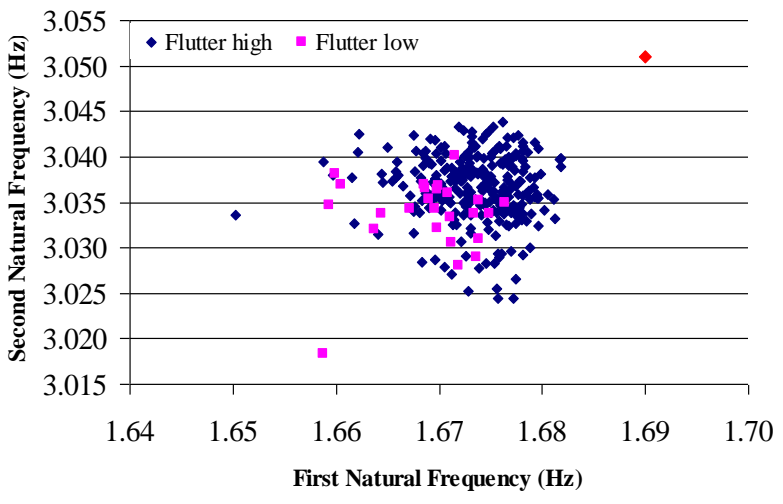

(a)

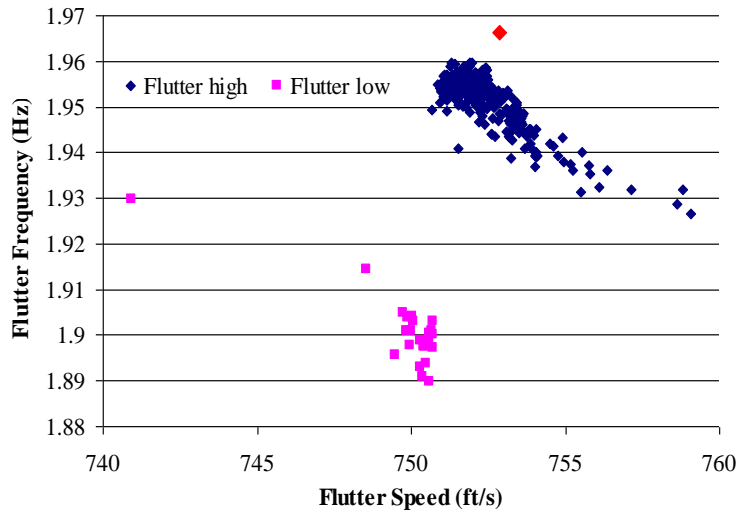

(b) 
Figure 14. Scatter plot of (a) first and second natural frequencies, (b) flutter frequency vs. flutter speed. The red diamond denotes the design conditions (fully clamped wing).

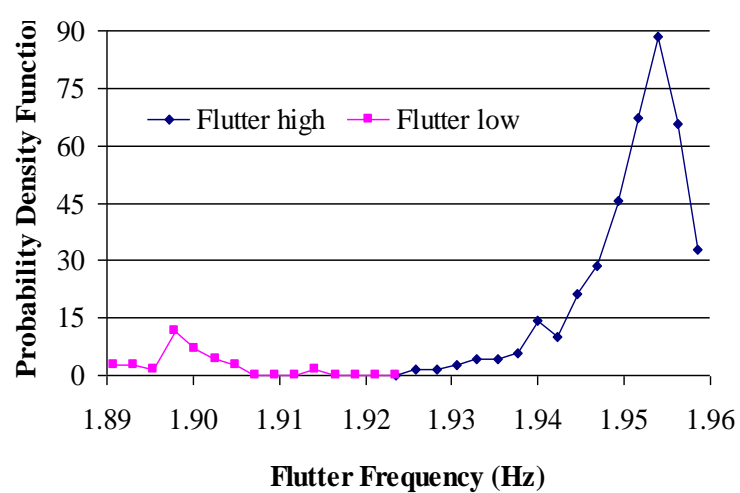

(a)

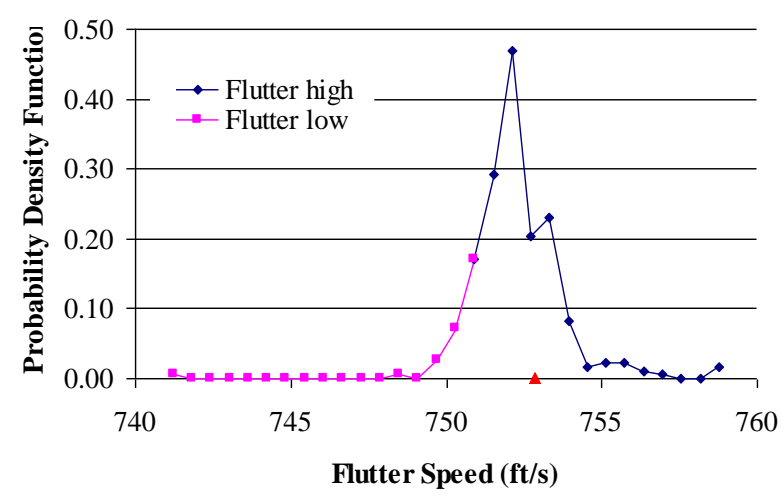

(b)

Figure 15. Probability density functions of (a) the flutter frequency and (b) the flutter speed. The red triangle denotes the design conditions (fully clamped wing).

It had been observed in a previous investigation of this wing [18] that the variations in flutter frequency and especially flutter speed were much larger than the corresponding variations of the natural frequencies resulting from uncertainty in the structure (not in the boundary conditions). Clearly, the results of Fig. 14 confirm this earlier finding to the case of uncertainty in the boundary conditions. It had further been observed [18] that two different flutter modes could occur and the probability density functions of Fig. 15 support again this finding here: the 300 flutter cases corresponding to uncertain boundary conditions can be separated into two groups, exhibiting either high or low flutter speed and frequency.

\section{Uncertain Coupling Between Substructures}

The modeling procedure described and assessed in the previous section can be extended to the consideration of uncertainty in the coupling between substructures such as the wing of Fig. 16. For simplicity, assume that there are only two substructures the dynamics of which will be represented by two sets of fixed boundaries mode shapes, $\Phi_{1}$ and $\Phi_{2}$, and two sets of constraint modes, $\boldsymbol{\Xi}_{1}$ and $\boldsymbol{\Xi}_{2}$. The subscripts 1 and 2 refer here to the inboard and outboard parts of the wing of Fig. 16, respectively. Then, contained in $\Phi_{1}$ are the mode shapes of the inboard wing clamped at both its root and the interface with the outboard one. Similarly, the modes in $\boldsymbol{\Phi}_{2}$ correspond to the outboard wing clamped at its interface with the inboard. The constraint modes, $\boldsymbol{\Xi}_{1}$ and $\boldsymbol{\Xi}_{2}$, are associated solely 
with the inboard-outboard interface if no flexibility/variability is considered at the inboard root. Otherwise, $\boldsymbol{\Xi}_{1}$ would also include the corresponding constraint modes.

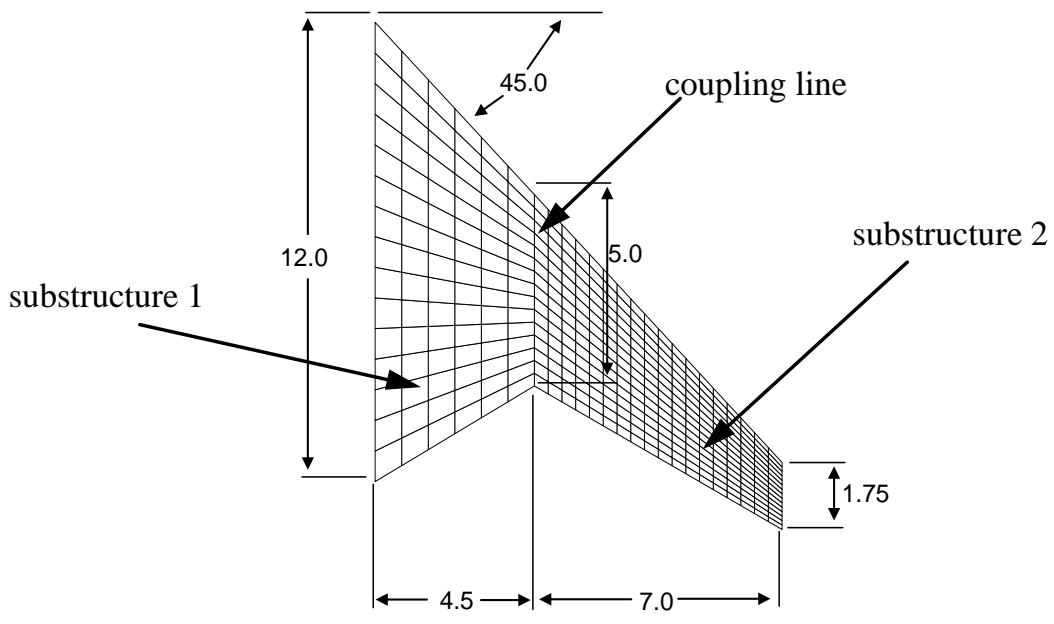

Figure 16. Wing example definition (dimensions in feet)

To obtain a reduced order model of the structure with flexible coupling between its substructures, denote first by $\boldsymbol{q}_{i}$ and $\boldsymbol{Y}_{i}, i=1,2$, the generalized coordinates associated with the modes and constraint modes of the two substructures. Further, introduce the expansion of the variables $\boldsymbol{Y}_{i}$ as

$$
\boldsymbol{Y}_{i}=\Psi_{i} \boldsymbol{u}_{i} \quad i=1,2
$$

where $\Psi_{i}$ denotes the eigenvector matrix of the boundary modes of structure $i$ obtained as in Eq. (19). Then, paralleling the developments of the previous sections, the stiffness and mass matrices of the reduced order model variables

$$
\boldsymbol{X}_{R O M}=\left[\begin{array}{llll}
\boldsymbol{q}_{1}^{T} & \boldsymbol{u}_{1}^{T} & \boldsymbol{q}_{2}^{T} & \boldsymbol{u}_{2}^{T}
\end{array}\right]^{T}
$$

are found to be

$$
\overline{\mathbf{K}}_{R O M}=\left[\begin{array}{cccc}
\overline{\boldsymbol{K}}_{R O M}^{q_{1} q_{1}} & \overline{\boldsymbol{K}}_{R O M}^{q_{1} u_{1}} & & \\
\overline{\boldsymbol{K}}_{R O M}^{u_{1} q_{1}} & \overline{\boldsymbol{K}}_{R O M}^{u_{1} u_{1}}+\hat{\boldsymbol{K}}_{R O M}^{u_{1} u_{1}} & & -\hat{\boldsymbol{K}}_{R O M}^{u_{1} u_{2}} \\
& & \overline{\boldsymbol{K}}_{R O M}^{q_{2} q_{2}} & \overline{\boldsymbol{K}}_{R O M}^{q_{2} u_{2}} \\
& -\hat{\boldsymbol{K}}_{R O M}^{u_{2} u_{1}} & \overline{\boldsymbol{K}}_{R O M}^{u_{2} q_{2}} & \overline{\boldsymbol{K}}_{R O M}^{u_{2} u_{2}}+\hat{\boldsymbol{K}}_{R O M}^{u_{2} u_{2}}
\end{array}\right]
$$

and 


$$
\overline{\boldsymbol{M}}_{R O M}=\left[\begin{array}{cccc}
\overline{\boldsymbol{M}}_{R O M}^{q_{1} q_{1}} & \overline{\boldsymbol{M}}_{R O M}^{q_{1} u_{1}} & & \\
\overline{\boldsymbol{M}}_{R O M}^{u_{1} q_{1}} & \overline{\boldsymbol{M}}_{R O M}^{u_{1} u_{1}} & & \\
& & \overline{\boldsymbol{M}}_{R O M}^{q_{2} q_{2}} & \overline{\boldsymbol{M}}_{R O M}^{q_{2} u_{2}} \\
& & \overline{\boldsymbol{M}}_{R O M} q_{2} & \overline{\boldsymbol{M}}_{R O M}^{u_{2} u_{2}}
\end{array}\right]
$$

with notations consistent with those introduced in the treatment of uncertain boundary conditions. That is,

$$
\left[\begin{array}{ll}
\overline{\boldsymbol{K}}_{R O M}^{q_{i} q_{i}} & \overline{\boldsymbol{K}}_{R O M}^{q_{i} u_{i}} \\
\overline{\boldsymbol{K}}_{R O M}^{u_{i} q_{i}} & \overline{\boldsymbol{K}}_{R O M}^{u_{i} u_{i}}
\end{array}\right]=\boldsymbol{T}_{2}^{(i) T} \boldsymbol{T}_{1}^{(i) T} \overline{\boldsymbol{K}}_{p h y s}^{(i)} \boldsymbol{T}_{1}^{(i)} \boldsymbol{T}_{2}^{(i)}
$$

where $\overline{\boldsymbol{K}}_{\text {phys }}^{(i)}$ is the stiffness matrix of the free component $i$ and

$$
\boldsymbol{T}_{1}^{(i)}=\left[\begin{array}{cc}
\boldsymbol{\Phi}_{i} & \boldsymbol{\Xi}_{i} \\
\mathbf{0} & \boldsymbol{I}
\end{array}\right] \quad \boldsymbol{T}_{2}^{(i)}=\left[\begin{array}{cc}
\boldsymbol{I} & \mathbf{0} \\
\mathbf{0} & \boldsymbol{\Psi}_{i}
\end{array}\right]
$$

and similarly for the mass matrix partitions. Further, assuming as in Eq. (24), one has

$$
\hat{\boldsymbol{K}}_{R O M}^{u_{i} u_{j}}=\Psi_{i}^{T} \hat{\boldsymbol{K}}_{\text {phys }} \Psi_{j}
$$

where $\hat{\boldsymbol{K}}_{\text {phys }}$ is the coupling stiffness joining the two substructures at their common interface. In parallel with the discussion of the previous section, it is assumed here that

$$
\hat{\boldsymbol{K}}_{\text {phys }}=k\left\lfloor\overline{\boldsymbol{K}}_{\text {phys }}^{B_{1} B_{1}}+\overline{\boldsymbol{K}}_{\text {phys }}^{B_{2} B_{2}}\right\rfloor
$$

where the notation $B_{i} B_{i}$ is introduced here to specify the side of the common boundary (i.e. substructure $i$ ). So, $\hat{\boldsymbol{K}}_{\text {phys }}$ is built from the sum of the physical stiffnesses of the two substructures at their interface nodes.

This approach has been demonstrated on the wing of Fig. 16. Given the small number of nodes at its interface, all constraint modes were kept and 20 clamped modes were taken for each substructure. The behavior of the mean flexible-interface model was first analyzed and shown in Fig. 17 is the convergence, as $k$ increases, of the first five natural frequencies of the system (Fig. 17(a)) and of their corresponding mode shapes (Figs 17(b) and 18) to their counterparts for the single cantilevered wing. Note the similarity of behavior with the plots of Fig. 3 .

Given the cantilevered nature of the wing of Fig. 16, it was expected that the introduction of flexibility at the coupling line would affect the mode shapes not only near that line but also near the tip. The mode shape projection remainders (or vector rejections) $\Delta \varphi_{j}$, see Eq. (28) and Fig. 19, confirm this expectation somewhat for mode 2 but definitely for modes 3 and 4 while mode 1 is only affected at the coupling line. 


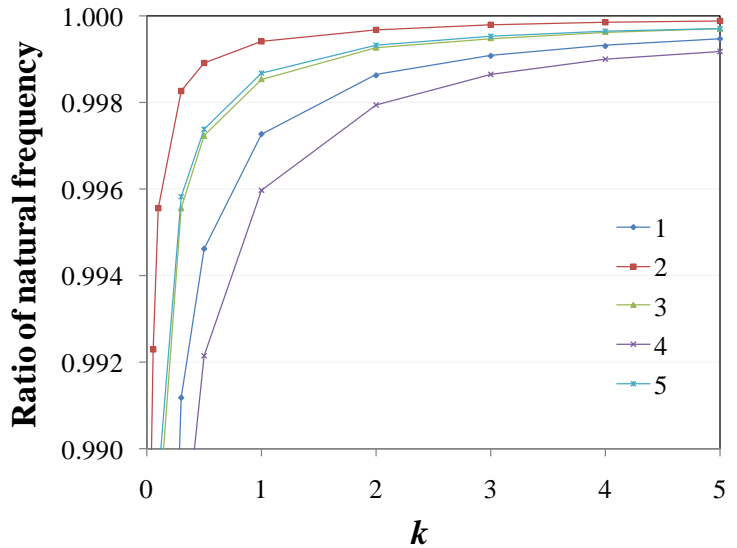

(a)

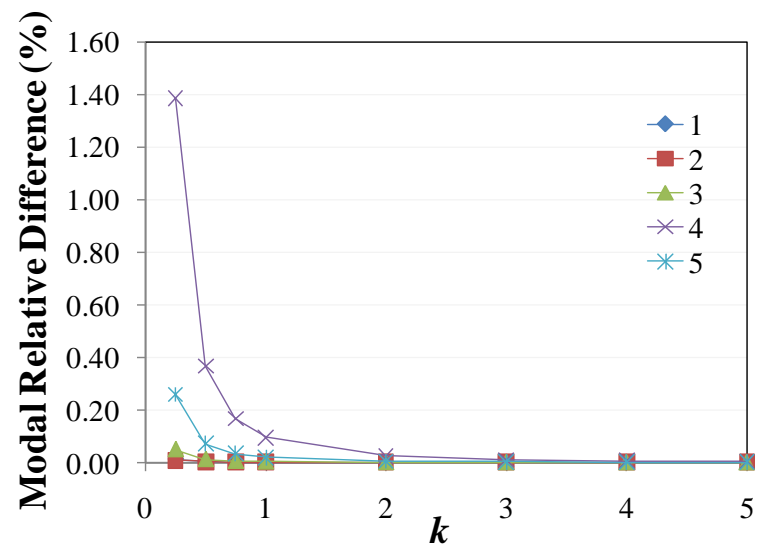

(b)

Figure 17. Convergence of the first five (a) natural frequencies and (b) mode shapes of the flexibility coupled wing as a function of $k$ to their $k=\propto$ counterparts, all boundary modes included.

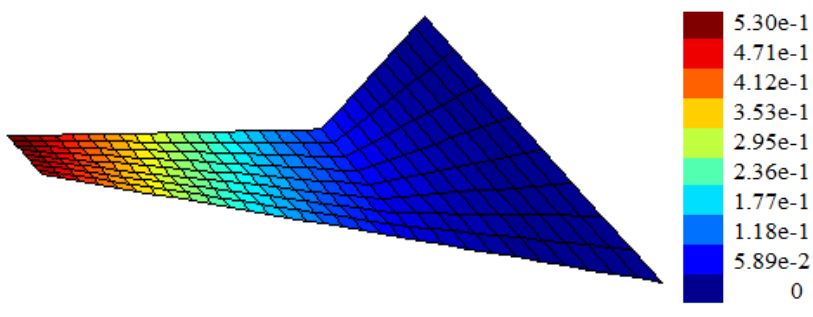

(a)

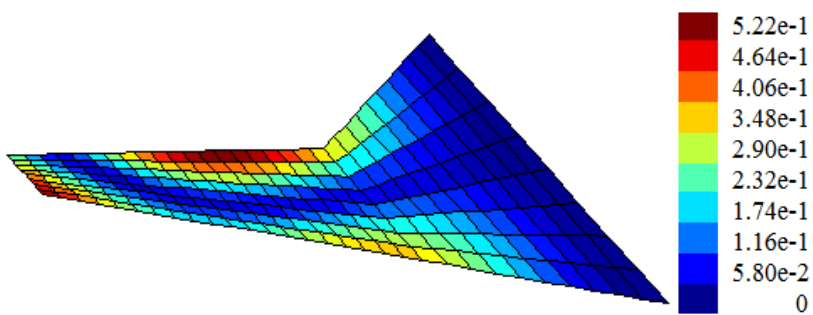

(c)

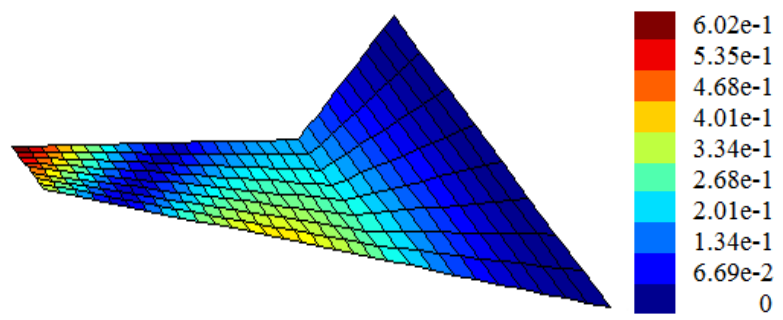

(b)

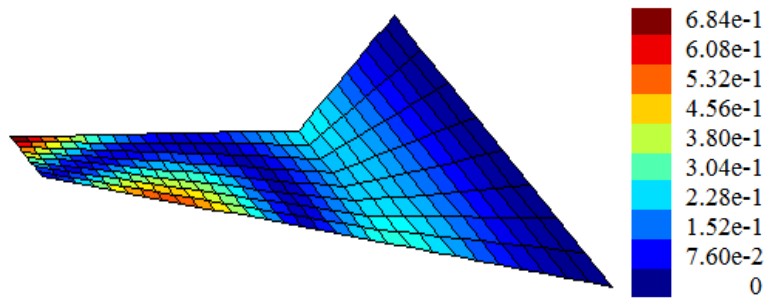

(d)

Figure 18. Mode shape magnitudes of the wing model of Fig. 16 with flexible coupling line $k=0.25$. (a),(b),(c),(d) modes 1, 2, 3, and 4.

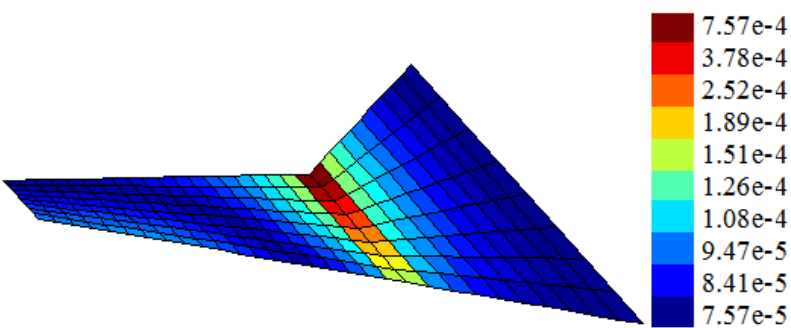

(a)

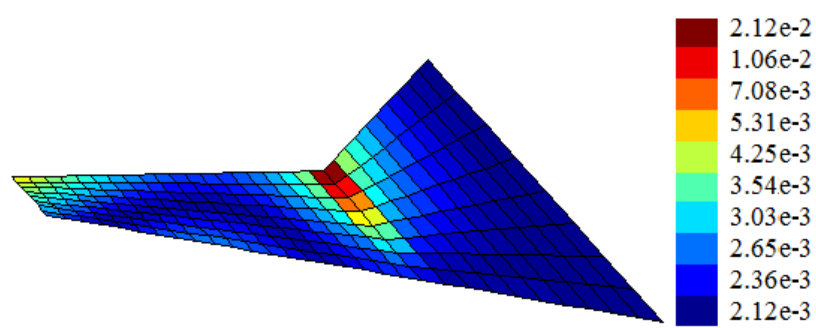

(b) 


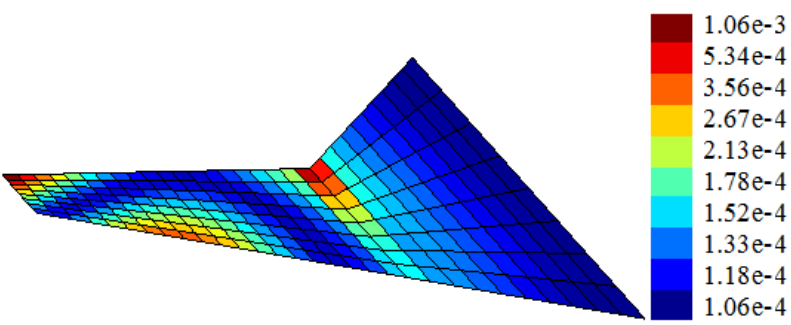

(c)

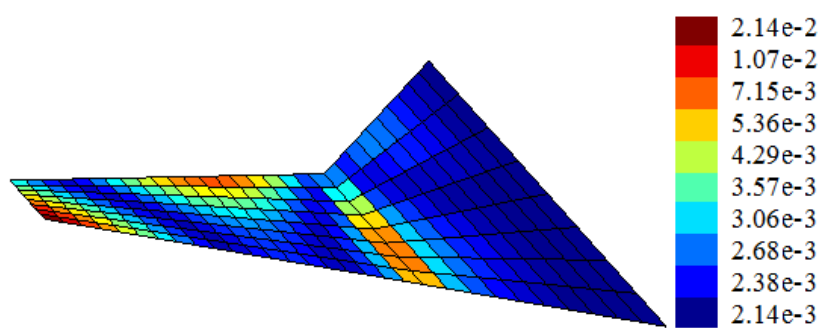

(d)

Figure 19. Remainder $\Delta \boldsymbol{\varphi}_{j}$ of the projection, or vector rejection, of the flexible coupling line modes on their rigid boundary counterparts. (a),(b),(c),(d) modes 1, 2, 3, and 4. Wing model of Fig. 16 with $k=0.25$.

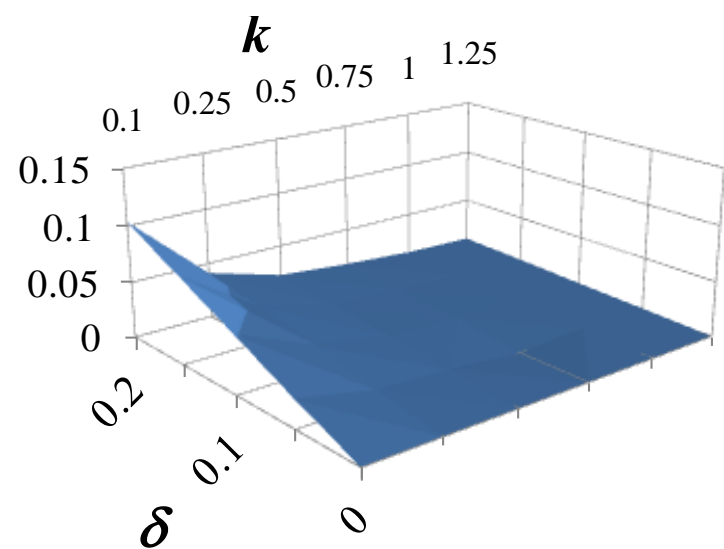

(a)

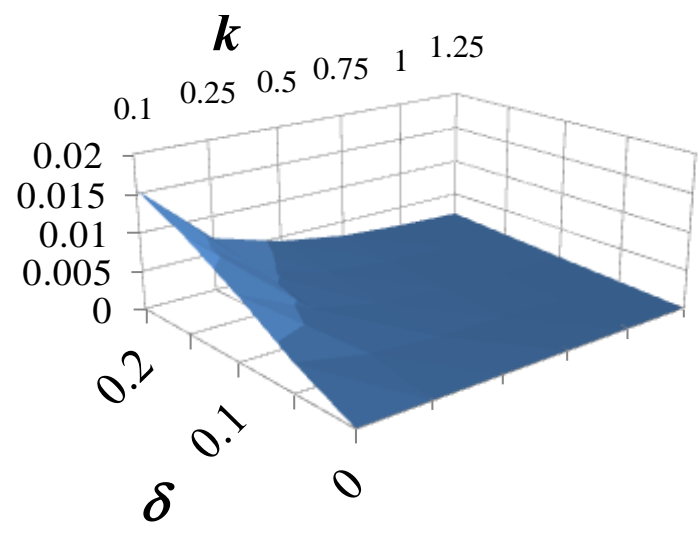

(b)

Figure 20. Coefficients of variation (in \%) of the first two natural frequency vs. $k$ and $\delta$, all boundary modes, 20 clamped modes each substructure, wing model of Fig. 16.

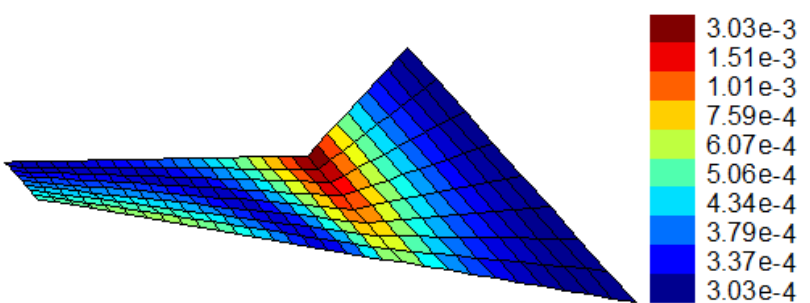

(a)

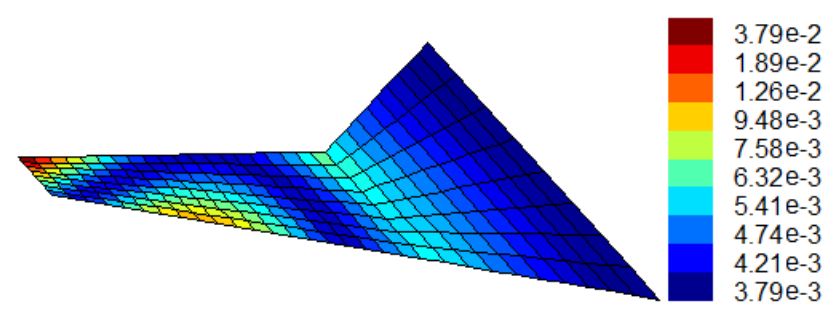

(c)

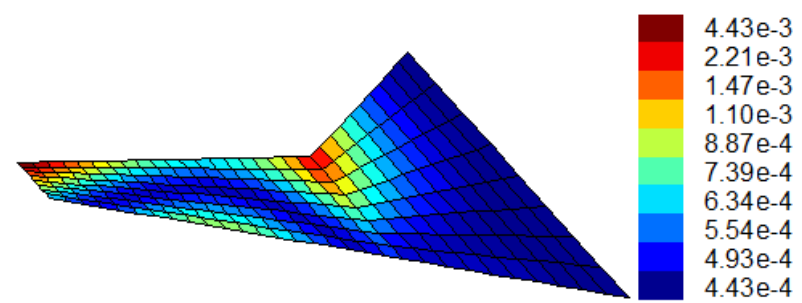

(b)

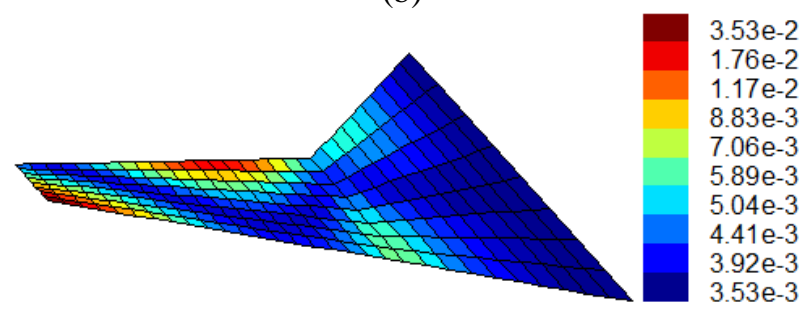

(d)

Figure 21. Standard deviation of the mode shapes of the wing model of Fig. 16 with flexible coupling line. $k=0.25, \delta=0.1$. (a),(b),(c),(d) modes $1,2,3$, and 4 . 
Uncertainty in the coupling of the two substructures was introduced next and the effects of varying the parameters $k$ and $\delta$ on the coefficient of variation of the natural frequencies were first assessed. The results, see Fig. 20, demonstrate the same monotonic behavior with respects to $k$ and $\delta$ which had been observed with the clamped plate, Fig. 8. Further, an analysis of the mode shapes, see Fig. 21, confirms the strong correlation between the standard deviation of these modes and the remainders $\Delta \varphi_{j}$. Since the first mode variability is predominantly along the coupling line, the boundary energy metric $E_{B C}$ of Eq. (28) (with the same matrix $A_{B C}$ as before) applied to this mode, with $\boldsymbol{X}_{\text {phys }}^{B}$ the difference of modal values across the coupling line, can be introduced for the estimation of the parameters $k$ and $\delta$, see Fig. 22 for its mean and standard deviation. As in the clamped plate example, it is seen from these plots that the parameter $k$ relates again strongly to the mean of the boundary energy while its variance then permits the estimation of the dispersion parameter $\delta$.

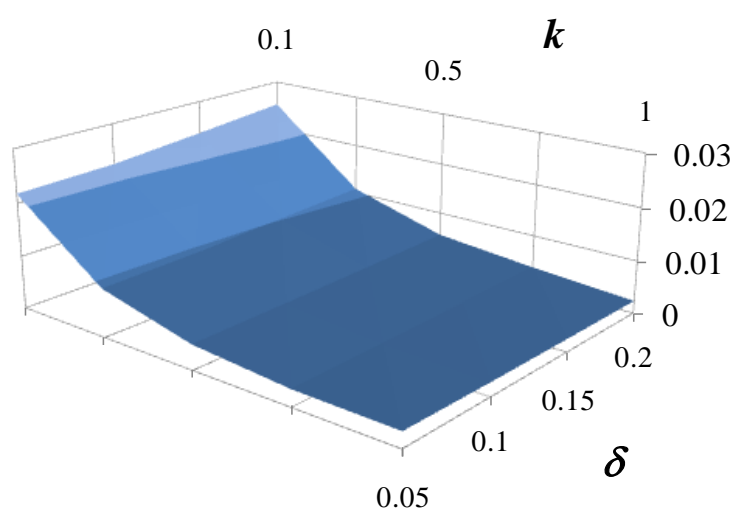

(a)

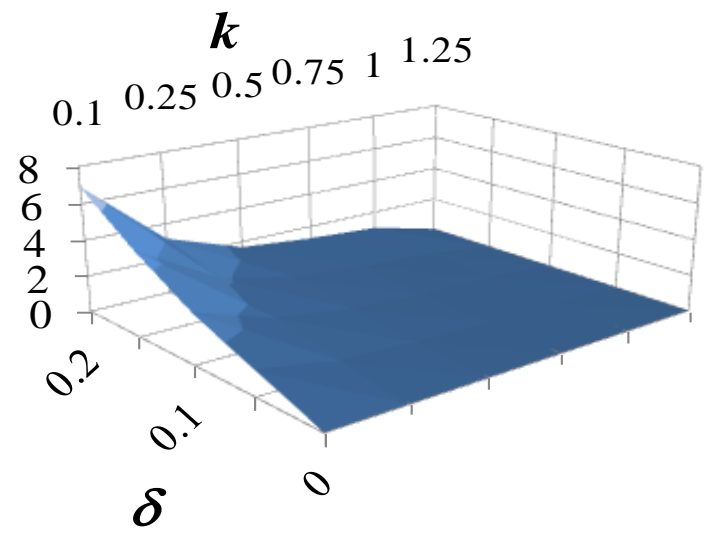

(b)

Figure 22. Mean and coefficient of variation (in \%) of $E_{B C}$ first mode deformations, vs. $k$ and $\delta$, all boundary modes, 20 clamped modes each substructure, wing model of Fig. 16.

\section{Conclusions}

The focus of this investigation was on the formulation and validation of a novel approach for the inclusion of uncertainty in the modeling of the boundary conditions of linear structures and of the coupling between linear substructures. The three steps of the approach are: (i) the determination of a mean structural dynamic model including boundary condition/coupling flexibility, (ii) the introduction of uncertainty in the mean model, and (iii) the estimation of the flexibility and uncertainty parameters of the model. 
A Craig-Bampton substructuring approach was adopted for the formulation of the mean model with boundary condition/coupling flexibility. This flexibility was implemented through the introduction of a finite stiffness matrix $\hat{\boldsymbol{K}}_{R O M}^{u u}$ expressed in terms of the physical stiffness matrix of the structure at its boundary, see Fig. 2 and Eq. (24). Note that differences between the assumption of Eq. (24) and the actual behavior of the structure's boundary add to the existing model uncertainty to be accounted for.

The simulation of uncertainty was addressed using the nonparametric modeling approach first because it addresses model and parameter uncertainties, both of which are expected to be present. The inclusion of model uncertainty permits in particular to account for the differences between the actual behavior of the structure's boundary and the assumption of Eq. (24). A second advantage of the nonparametric approach is its computational convenience as random matrices $\breve{\boldsymbol{K}}_{R O M}^{u u}$ are readily generated using the algorithm of Eqs (2)-(5) or Fig. 1.

Each of the above steps, i.e. the mean model construction and the nonparametric approach, introduces one parameter in the problem, i.e. $k$ to represent the boundary flexibility in the mean model and $\delta$ for the uncertainty characterization. The estimation of these parameters could be performed from global variables, e.g. from the mean and standard deviation of the first natural frequency, but they might then be affected by the presence of uncertainty on other aspects of the structure (other boundary/coupling, uncertain material/geometrical properties, etc.) Accordingly, it was proposed here to estimate $k$ and $\delta$ using measurements performed on the uncertain boundary. In fact, the value of $k$ was readily shown to correlate very strongly to the mean energy on the boundary, see Eq. (28), while $\delta$ exhibited a similar relationship with the standard deviation of this energy.

These developments permitted the flutter analysis of the Goland+ wing of Fig. 11 with uncertain boundary conditions. It was observed in particular that the resulting level of uncertainty on the flutter boundary was significantly larger than on the natural frequencies and thus is of practical importance.

Finally, the consideration of uncertainty in the coupling between substructures was formulated in a similar manner to that of the boundary conditions through a Craig-Bampton modeling approach of all connecting substructures. The application of these concepts was demonstrated on the 2-piece wing model of Fig. 16.

\section{Acknowledgements}

The authors wish to express their appreciation to ZONA Technology for the use of both ZAERO and the two wing models. 


\section{References}

[1] Soize, C., 2005, "A Comprehensive Overview of a Non-Parametric Probabilistic Approach of Model Uncertainties for Predictive Models in Structural Dynamics," Journal of Sound and Vibration, Vol. 288, pp. 623652.

[2] Ghanem, R., and Spanos, P.D., 1991, Stochastic Finite Elements: A Spectral Approach, Springer-Verlag, New York.

[3] LeMaitre, O.P., and Knio, O.M., 2010, Spectral Methods for Uncerainty Quantification with Applications to Computational Fluid Dynamics, Springer, Heidelberg.

[4] Mace, R., Worden, W., and Manson, G., 2005, "Uncertainty in Structural Dynamics," Journal of Sound and Vibration, Vol. 288, No. 3, pp. 423-429.

[5] Schueller, G. I., 2005, "Uncertainties in Structural Mechanics and Analysis-Computational Methods," Computer and Structures, Vol. 83, No. 14, pp. 1031-1150.

[6] Avalos, J., Mignolet, M.P., and Soize, C., 2009, "Response of Bladed Disks with Mistuned Blade-Disk Interface," Proceedings of the IGTI Turbo Expo'09, Orlando, Florida, Jun. 8-12, ASME Paper GT-2009-59580.

[7] Schwarz, B., Richardson, M., and Formenti, D.L., 2007, "FEA Model Updating Using SDM," Presented at the International Modal Analysis Conference, IMAC XXV, Orlando, Florida.

[8] Elishakoff, I., Fang, J., 1995, "Diagnosis of Local Uncertain Modifications in the Boundary Conditions of a Rectangular Plate via Convex Modeling," Computational Methods in Applied Mechanics and Engineering, Vol. 124, pp. 303-319.

[9] Huang, B., Li, Q.S., Shi, W.H., and Wu, Z., 2007, "Eigenvalues of Structures with Uncertain Elastic Boundary Restraints," Applied Acoustics, Vol. 68, pp. 350-363.

[10] Blanze, C., and Rouch, P., 2005, "Analysis of Structures with Stochastic Interfaces in the Medium-Frequency Range,” Journal of Computational Acoustics, Vol. 13, No. 4, pp. 711-729.

[11] Soize, C., and Chebli, H., 2003, "Random Uncertainties Model in Dynamic Substructuring Using a Nonparametric Probabilistic Model," Journal of Engineering Mechanics, pp. 449-457.

[12] Soize, C., "Random Matrix Theory for Modeling Uncertainties in Computational Mechanics," Computer Methods in Applied Mechanics and Engineering, Vol. 194, 2005, pp. 1333-1366.

[13] Mignolet, M.P., and Soize, C., "Nonparametric Stochastic Modeling Of Linear Systems With Prescribed Variance Of Several Natural Frequencies," Probabilistic Engineering Mechanics, Vol. 23, Nos. 2-3, Apr.-Jul. 2008, pp. 267-278.

[14] Craig, R.R., and Kurdila, A.J., Fundamentals of Structural Dynamics, $2^{\text {nd }}$ ed., John Wiley \& Sons, New Jersey, 2006, Chap. 17.

[15] Craig, R.R., and Bampton, M.C.C., "Coupling of Substructures for Dynamic Analysis," AIAA Journal, Vol. 6, No. 7, 1968, pp. 1313-1319.

[16] Benfield, W.A., and Hruda, R.F., "Vibration Analysis of Structures by Component Mode Substitution," AIAA Journal, Vol. 9, No. 7, 1971, pp. 1255-1261.

[17] Rubin, S., "Improved Component-Mode Representation for Structural Dynamic Analysis," AIAA Journal, Vol. 13, No. 8, 1975, pp. 995-1006.

[18] Mignolet, M.P., and Chen, P.C., "Aeroelastic Analyses with Uncertainty in Structural Properties," Proceeding of the AVT-147 Symposium: Computational Uncertainty in Military Vehicle Design, Athens, Greece, 2007, Dec. 3-7. [19] Beran, P., Khot, N., Eastep, F., Snyder, R., and Zweber, J., "Numerical analysis of store-induced limit-cycle oscillation," Journal of Aircraft, Vol. 41, No. 6, 2004, pp. 1315-1326.

[20] Kurdi, M., Lindsley, N, and Beran, P., "Uncertainty Quantification of the Goland+ Wing's Flutter Boundary," Presented at the AIAA Atmospheric Flight Mechanics Conference and Exhibit, Aug. 20 - 23, 2007, Hilton Head, S.C., AIAA Paper AIAA-2007-6309. 